\title{
Common metabolic networks contribute to carbon sink strength of sorghum internodes: implications for bioenergy improvement
}

\author{
Yin $\mathrm{Li}^{i^{*}} \mathbb{0}, \mathrm{Min} \mathrm{Tu}^{1}$, Yaping Feng ${ }^{1}$, Wenqin Wang ${ }^{2}$ and Joachim Messing ${ }^{1 *}$
}

\begin{abstract}
Background: Sorghum bicolor (L.) is an important bioenergy source. The stems of sweet sorghum function as carbon sinks and accumulate large amounts of sugars and lignocellulosic biomass and considerable amounts of starch, therefore providing a model of carbon allocation and accumulation for other bioenergy crops. While omics data sets for sugar accumulation have been reported in different genotypes, the common features of primary metabolism in sweet genotypes remain unclear. To obtain a cohesive and comparative picture of carbohydrate metabolism between sorghum genotypes, we compared the phenotypes and transcriptome dynamics of sugar-accumulating internodes among three different sweet genotypes (Della, Rio, and SIL-05) and two non-sweet genotypes (BTx406 and R9188).

Results: Field experiments showed that Della and Rio had similar dynamics and internode patterns of sugar concentration, albeit distinct other phenotypes. Interestingly, cellulose synthases for primary cell wall and key genes in starch synthesis and degradation were coordinately upregulated in sweet genotypes. Sweet sorghums maintained active monolignol biosynthesis compared to the non-sweet genotypes. Comparative RNA-seq results support the role of candidate Tonoplast Sugar Transporter gene (TST), but not the Sugars Will Eventually be Exported Transporter genes (SWEETS) in the different sugar accumulations between sweet and non-sweet genotypes.

Conclusions: Comparisons of the expression dynamics of carbon metabolic genes across the RNA-seq data sets identify several candidate genes with contrasting expression patterns between sweet and non-sweet sorghum lines, including genes required for cellulose and monolignol synthesis (CesA, PTAL, and CCR), starch metabolism (AGPase, SS, SBE, and G6P-translocator SbGPT2), and sucrose metabolism and transport (TPP and TST2). The common transcriptome features of primary metabolism identified here suggest the metabolic networks contributing to carbon sink strength in sorghum internodes, prioritize the candidate genes for manipulating carbon allocation with bioenergy purposes, and provide a comparative and cohesive picture of the complexity of carbon sink strength in sorghum stem.
\end{abstract}

Keywords: Bioenergy, RNA-seq, Gene expression, Transcriptome analysis, Carbon partitioning, Sugar accumulation, Internode, Sorghum

\section{Introduction}

A characteristic feature of vascular plants is that $\mathrm{CO}_{2}$ is fixed by photosynthesis in source leaves and then transported to and utilized by different sink organs for growth. During this process, three key factors can affect

\footnotetext{
*Correspondence: y1737@waksman.rutgers.edu; messing@waksman.rutgers.edu

${ }^{1}$ Waksman Institute of Microbiology, Rutgers, The State University of New Jersey, Piscataway, NJ 08854, USA

Full list of author information is available at the end of the article
}

source-to-sink relationship: (i) photosynthesis capacity that determines carbon availability; (ii) sugar transportation; (iii) carbon utilization and storage at sink organs [1]. During plant growth and development, sink organs/ tissues are dynamic [2, 3]. For example, immature leaves and shoot apical meristems are sink organs in vegetative stages while developing flowers and seeds become sinks in reproductive stages. Therefore, the abilities of sink organs to obtain, utilize, and store carbon (so-called 'sink strength') are dynamic and tightly controlled $[4,5]$. 
Moreover, the distribution of carbon utilization/storage (carbon allocation) within a sink tissue is well coordinated. The $\mathrm{C} 4$ grasses include important bioenergy crops, such as maize, sorghum, switchgrass, and sugarcane, and serve as the most-significant plant source of carbohydrates and bioethanol [6]. Among these C4 crops, Sorghum bicolor is an excellent example for studying carbon allocation, because sweet sorghum varieties have two sink organs, seeds, and stem $[7,8]$. While a significant portion of carbon reserves are in cell wall components, large amounts of soluble sugars (primarily sucrose) and starch accumulate in sorghum stems after flowering. Thus, this feature makes sweet sorghum an interesting model to study carbon partitioning and sugar accumulation for other bioenergy crops like sugarcane $[9,10]$. In addition, sweet sorghum can accumulate considerable amount of starch in the internode [11] and has differential expression patterns of the cell wall-related genes compared to non-sweet genotypes [12], indicating that the distribution of carbon utilization within sweet sorghum internodes may be redirected to establish sink strength. Also, sorghum is an emerging bioenergy crop with multiple advantages: (i) a 730-Mb diploid genome and several reference assemblies with great synteny to maize and sugarcane [13-16]; (ii) good tolerance to several abiotic stresses and desirable agronomical features, such as the stay-green trait $[7,17,18]$; (iii) rich genetic resources [19], such as several EMS resources [20-22]; (iv) ability to be transformed and genome-edited [23, 24]; (iv) potential in phytoremediation of soil pollution [25].

Knowledge of sorghum stem sugar accumulation has accrued from genetics, physiology, molecular biology, and omics studies. Sucrose starts to increase after internode elongation, with a dramatic accumulation from anthesis to the first 2 weeks post-anthesis $[12,26]$. Population genetics studies revealed that stem sugar yield is determined by three factors: stem juiciness, stem biomass-related traits, and sugar concentrations of the juice, the first two affecting juice volume/weight $[27,28]$. Sorghum stem juiciness is largely controlled by a single gene, named Dry culms (D) [29], which encodes an NAC transcription factor controlling programmed cell death of stem parenchyma cells, thereby affecting secondary cell wall compositions [30-33]. While quantitative trait loci (QTL) associated with sugar-related traits have been reported in sorghum [34-39], the molecular mechanism regulating stem sugar concentrations remains unclear. Physiology results using radiolabeling and dye transport approaches suggest that sucrose may be transported to storage parenchyma via apoplasmic and/or symplasmic routes [40-43].

Carbohydrates are stored in sorghum stems in three significant forms, sucrose in vacuoles, starch in plastids, and lignocellulosic cell wall biomass [26]. The sucrose in vacuoles could be related to several sugar transporters, such as Sucrose Transporters (SUTs), Tonoplast Sugar Transporters (TSTs), and Sugars Will Eventually be Exported Transporters (SWEETs). The expression profiles of these transporters have been examined in sweet and grain genotypes [12, 42-46], suggesting $S b T S T 2$ as a candidate gene for stem sugar difference between sweet and grain sorghum lines [46]. The sorghum SWEETs fell into the four phylogenetically defined clades, in which evidence of phylogeny-function correlation has been shown in several species [47-54]. Starch synthesis requires a suite of well-characterized enzymes and transporters (reviewed previously in $[55,56])$, including ADP-glucose pyrophosphorylase (AGPase), soluble starch synthase (SS), granule-bound starch synthase (GBSS), starch branching enzyme (SBE), starch debranching enzyme (DBE)/isoamylase (ISA), and glucose-6-phosphate translocators (GPT) that fuel starch synthesis with glucose1-phosphate (G1P) [57]. Starch is degraded by a set of kinases and hydrolases, including glucan-water dikinase (GWD), phosphor-glucan-water dikinase (PWD), $\alpha$ - and $\beta$-amylase (AMY and BAM, respectively) and disproportionating enzyme (DPE) [58-60].

Plant cell walls include primary and secondary cell wall (PCW and SCW, respectively). PCW, mainly composed of cellulose, hemicellulose, and pectin, exists in all plant cell types and is tensile to yield to cell expansion and turgor pressure. SCW, mainly composed of lignin, crosslinked with cellulose and hemicellulose, exists in specific cell types to provide mechanical support and serve as a defensive barrier. Cellulose, as the most abundant structural polysaccharide in plant cell wall, is synthesized by cellulose synthases that are encoded by CesA gene family [61, 62], of which two phylogenetic groups are responsible for PCW and SCW biosynthesis, respectively [63]. Hemicelluloses are branched hetero-carbohydrate polymers synthesized by cellulose synthase-like (Csl) enzymes. Lignin is a complex heteropolymer crosslinked from three monolignins, namely $p$-coumaryl $(\mathrm{H})$, coniferyl $(\mathrm{G})$, and sinapyl $(\mathrm{S})$ alcohols [64]. Ten major gene families required for monolignol biosynthesis have been well studied in sorghum at the genome level [65], namely, phenylalanine ammonia-lyase (PAL), cinnamate 4-hydroxylase $(\mathrm{C} 4 \mathrm{H})$, 4-coumarate:CoA ligase (4CL), hydroxycinnamoyl-transferase (HCT), 4-coumarate 3-hydroxylase $(\mathrm{C} 3 \mathrm{H})$, cinnamyl-CoA reductase (CCR), cinnamyl alcohol dehydrogenase (CAD), caffeic acid $O$-methyltransferase (COMT), caffeoyl-coenzyme A 3-O-methyltransferase (CCOAOMT), and ferulate 5 -hydroxylase $(\mathrm{F} 5 \mathrm{H})$. Seven out of the ten enzymes have been structurally and biochemically investigated (see "Methods"). Three Brown midrib (Bmr) loci are known to encode enzymes of monolignol biosynthesis [66]. 
Recent studies of sorghum stem sugar accumulation have expanded to gene and genome levels, including comparisons between grain and sweet sorghum using whole-genome re-sequencing $[67,68]$ and mRNA and small RNA transcriptome analyses [69-72]. RNAseq data of sugar-accumulating internodes have been reported in three sweet sorghum genotypes [12, 26, 45]. McKinley et al. focused on the expression of biosynthetic genes of cell wall components across reproductive development in sweet sorghum Della [26] and expanded their analysis to starch metabolism, demonstrating the upregulation of starch-metabolic genes and the tendency of higher starch contents in sweet genotypes [11]. Mizuno et al. [45] focused on SWEET transporters in Japanese sweet sorghum SIL-05. Li et al. integrated a time-series of transcriptome and metabolome data of sorghum stems [12] and revealed that: (1) carbon sink strength in stem is related to the coordination of several primary metabolic pathways, and (2) the sucrose signal, trehalose-6-phosphate (T6P), may be involved in stem sugar accumulation. However, important biological questions remain unanswered: (1) Are the afore-mentioned candidate pathways/genes specific for a given sweet genotype? (2) What are the common expression features of primary metabolism and sugar transport among different sweet sorghum lines? Several previous studies either focused on a particular aspect of carbohydrate metabolism or a gene family $[11,26,45]$. A single omics data set is insufficient to answer the above questions and is unable to provide a comprehensive and cohesive molecular view of carbon partitioning and sink strength in sorghum stem. To address these issues, we performed a detailed comparative transcriptome analysis among the three RNAseq data sets with a focus on sucrose-related metabolic pathways and also characterized the sugar accumulation dynamics of the sweet sorghums Rio and Della. Here, we report that the three sweet genotypes have similar expression profiles in key genes involved in carbon utilization pathways and sucrose transport, highlighting the reliable candidate genes from the TST family, CesA family, and starch-metabolic and monolignol biosynthetic pathways with common expression profiles among sweet genotypes. We question the involvement of the potential SWEET candidates based on their non-consensus expression patterns and phylogeny-function correlation.

Our analysis allows us to propose a common metabolic network for carbon partitioning and sink establishment in sweet sorghum internodes. The common network implies that: (1) the major carbon reserves reflected by the analyzed primary metabolic pathways and sucrose transportation jointly contribute to the sink strength of stem tissue; (2) the activities of the primary metabolic pathways reflect the distribution of carbon utilization in the stem; (3) carbon allocation could be changed by manipulating the identified candidate genes and, hence, the corresponding carbon reserves to improve stem carbohydrate compositions for bioenergy purposes.

\section{Methods \\ Plant materials and field experiments}

Three sweet sorghum genotypes, Rio, Della, and SIL-05, were used (Additional file 1). Rio (PI 651496) is developed from a cross of Rex (PI 641835) and Manawan (PI 152959) [73], whereas Della is developed from a cross of Dale (PI 651495) and ATx622 [74]. SIL-05 is a Japanese sweet sorghum line developed from a cross of BR504 and Brown Native by Kyushu, Okinawa Agricultural Research Center of NARO (National Agricultural and Food Research Organization. http://www.naro.affrc.go. jp/patent/breed/0500/0509/001471.html).

Rio and Della were obtained from the United States Department of Agriculture National Plant Germplasm System (USDA-NPGS) and phenotyped at the Waksman experimental field (Piscataway, NJ) in 2018. SIL05 was not phenotyped due to unavailable seeds from USDA-NPGS. Rio and Della were grown in each 8-row plot, with each row containing ten germinated plants. Only the six central plants per row from the six central rows were used for phenotyping to minimize border effect. Plant height, number of above-ground internode, days to flower, and internode total sugar concentration were recorded at six stages, including anthesis, 10, 18, 22,32 , and 38 days after flowering (DAF) to capture the sugar concentration dynamics. Internode fresh weight and dry weight were measured at five stages (from 10 to 38 DAF). Dry weight was measured after internode samples were dried at $65{ }^{\circ} \mathrm{C}$ for $96 \mathrm{~h}$. As an indicator of juice volume, internode water content was calculated as previously described [33]. The total sugar concentration of internode-extracted juice was measured by Brix [75]. All internode samples were collected in the field at 9:00-11:00 AM and stored on ice. After transferring samples back to the laboratory, the juice was extracted immediately.

\section{RNA-seq data analysis}

Three RNA-seq data sets were used (Additional file 2). The first data set is the transcriptomes of sugar-accumulating internodes from a conversion line R9188 and its two parents Rio and BTx406 collected at flag leaf stage, flowering and 10 and 15 days after flowering (designated as T1, T2, T3 and T4, respectively) [12]. The dwarf inbred line R9188 was developed from the BTx406/Rio cross followed by one backcross to Rio and contains the early flowering and dwarf loci introgressed from BTx406 [76]. RNA was extracted from the pooled tissues from upper internodes 
of Rio, BTx406 and R9188 (internode 2, 3, and 4, numbered from top to bottom) as described elsewhere [12].

The second RNA-seq data set is the transcriptomes of Della internodes collected from eight developmental stages $(29,16$, and 7 days before anthesis, anthesis, and $11,25,43$, and 68 days after anthesis, designated as A-29, A-16, A-7, A0, A11, A25, A43, and A68, respectively) [26]. Particularly, Della stem was fully mature at A-7 and the grains reached a soft dough stage at A25 and became completely mature before A43. RNA was extracted using the tenth internode of greenhouse-grown plants (numbered from bottom to top), while field grown Della in Texas, US had 14-15 internodes as previously described [26].

The third RNA-seq data set is the SIL-05 transcriptomes of internode, panicle, and leaf tissues at three stages, 1, 17, and 36 days after heading (1 DAH, $17 \mathrm{DAH}$, and 36 DAH, respectively) [45]. SIL-05 flowers between 1 and 17 DAH, and sucrose starts to accumulate in SIL-05 stem between 1 and $36 \mathrm{DAH}$ and can reach $18.9 \%$ in juice at $64 \mathrm{DAH}$. RNA was extracted from the corresponding internode from the leaf below flag leaf of SIL05 as described previously $[45,77]$.

The stages of the three RNA-seq data sets were aligned relative to anthesis and the stages over stem sugar accumulation were identified for each genotype (Fig. 1). To overcome the issues of lacking replicates (for dataset3) and unavailability of raw data (for dataset2), we took the following strategies for analysis (Additional files $3,4)$. (1) The raw data of data sets 1 and 3 were quality filtered and analyzed using the same pipeline. Reads were mapped to the sorghum reference genome (BTx623, Sbicolor_v2.1_255) using TopHat v2.0.14 (a maximum mismatch of $9 \mathrm{bp}$ and default settings for other parameters) [13, 78]. Read counts were calculated using 'HTseq' with uniquely mapped reads and RPKM values were calculated for SIL-05 [79]. (2) The dataset2 expression matrix using reads per kilobase of transcript per Million mapped reads (RPKM) were reversely calculated to the normalized average read counts for three replicates of each time point based on RPKM definition with the assumption that in Della, every gene has the same gene length as that in the genotypes from data sets 1 and 3 . Then, the normalized average read counts can be considered as an input of read count matrix without replicates for differential expression analysis. (3) To perform differential expression analysis for data sets 2 and 3 without replicates, the gene-wise dispersion of biological variation (using biological coefficient of variance, $\mathrm{BCV}$, as index) for sorghum internode tissues was calculated using the triplicates of dataset1 with "edgeR" [80]. The BCV matrix was used to identify differentially expressed genes in data sets 2 and 3 with "edgeR" using the following criteria: $q$ values $<0.05$ and $\log _{2}$ Fold Change $\left(\log _{2} \mathrm{FC}\right) \geq 1$. (4) To investigate the sample relationship across data sets, potential batch effects for the expression matrix between the three RNA-seq studies were minimized with quantile normalization using 'preprocessCore' as described in Additional file 4 [81, 82].

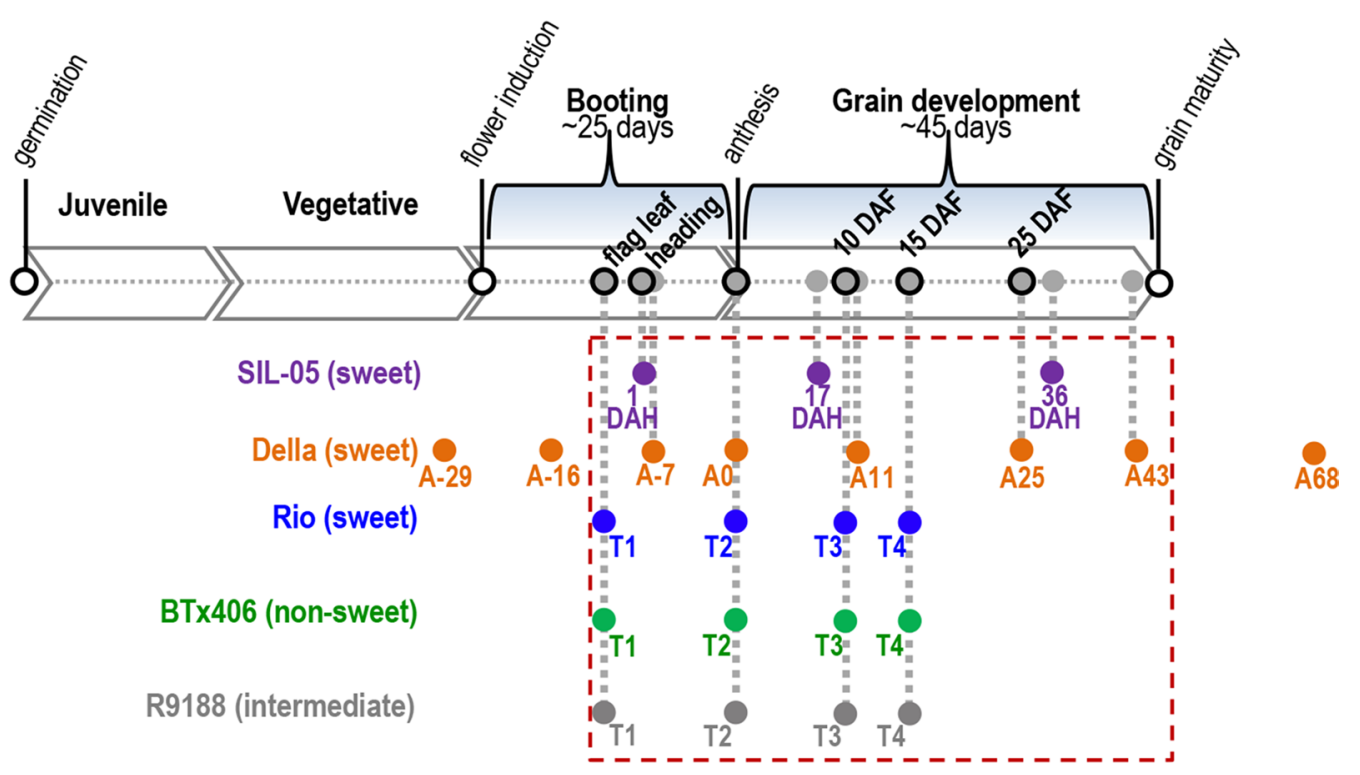

Fig. 1 Time-course alignment of the RNA-seq time points between sorghum genotypes. Duration of booting and grain development stages were according to the field observations for Rio and Della, and previous studies [12, 26, 45, 77]. The time points when the RNA-seq samples were collected are color-coded by genotypes and aligned to the developmental process of sorghum, with those samples related to stem sugar accumulation highlighted in the red broken box 
To identify common candidate genes associated with stem sugar accumulation in all the data sets, we investigated genes involved in primary metabolism and sugar transporters, and considered candidates using the following criteria. (1) Low-expression genes were excluded (maximum RPKM $\leq 5$ ), because the genes are annotated as encoding enzymes or transporters functioning in primary metabolic pathways that are responsible for major carbon reserves in sorghum stem. (2) To identify genes showing distinct expression trends between sweet and non-sweet genotypes, the genes should meet two criteria: (2A) differential expression at post-anthesis stages compared to anthesis or preanthesis stages in the sweet genotypes, but not in the non-sweet genotypes, or vice versa; (2B) an expression trend in Della and SIL05 similar to that in Rio, but contrasting to BTx406/R9188. To visualize the similar expression trends of selected candidate genes in sweet versus non-sweet comparison, heatmap of $\log _{2}$ fold change was used with the fold changes calculated using $\mathrm{RPKM}+0.1$ to avoid zero values.

\section{Identification of genes involved in primary metabolism and sugar transport}

Annotation information of genes potentially involved in cell wall metabolism, starch and sucrose metabolism, and glycolysis and sucrose transporter families (SUTs, SWEETs, and TSTs) was extracted from earlier literature and databases [83-89]. Detailed methods for gene annotation are in Additional file 4. All the gene annotation information is shown in Additional file 5.

\section{Phylogenetic analysis of SWEET gene family}

The 23 SWEETs reported previously were used for gene family analysis (Additional file 6). To re-confirm that these genes encode putative SWEETs, BLAST searches against sorghum genomes v2 and v3 followed by filtering using two MtN3 domain (Pfam: PF03083) were performed $[15,47]$. The deduced amino acid sequences of sorghum SWEETs were compared with rice and maize SWEET proteins; only primary or canonical transcripts were used. The rice and maize SWEETs were described previously [52-54]. SbSWEET nomenclature was according to Bhimidine et al. [46]. Sequence alignment was performed using MUSCLE and neighbor joining (NJ) phylogenetic trees were generated using MEGA v7 with JTT protein substitution model, pairwise deletion for gaps/missing data, and 1000-time bootstrap [90]. Sobic.003G038800 was not included in phylogenetic tree due to its two incomplete MtN3 domain (Additional file 7). Sorghum expression atlas and MOROKOSHI database were used to evaluate the spatio-temporal expression patterns of SWEETs [15, 84].

\section{Quantitative PCR validation}

Total RNA was extracted from the pooled samples of upper internodes (internodes 2, 3, and 4, numbered from top to bottom) for Rio, BTx406, and R9188, respectively, using TRIZOL and PureLink RNA extraction kit (Invitrogen). The samples were collected from the plants grown in a split-plot design and are the same samples used for RNA-seq of Rio/R9188/BTx406 as described previously [12]. The concentration and purity of the RNA were evaluated using a Nanodrop 2000 spectrophotometer. After cDNA synthesis with SuperScript III First Strand kit, real-time quantitative PCR (qPCR) was conducted with PowerUp SYBR Green mastermix (Thermo Fischer) using the ABI StepOne Plus Real-Time PCR system. Relative expression levels were calculated using the $\Delta \Delta C T$ method with Ubiquitin as the internal reference gene because of its stable expression determined by the RNAseq data [12]. All real-time qPCR primers are listed in Additional file 8.

\section{Results \\ Dynamics of internode sugar accumulation}

We compared the dynamics of internode sugar accumulation between Della and Rio, which had similar plant heights but differed in above-ground internode number (Fig. 2a, b). Both genotypes showed slight difference in days to flowering (Fig. 2c). Della and Rio showed similarities in internode sugar concentration dynamics in several aspects. First, the total sugar concentrations were markedly increased in both genotypes from anthesis to 38 DAF (from $\sim 9$ to $\sim 18 \%$ in Della and from $\sim 12$ to $\sim 19 \%$ in Rio; Fig. $2 d$ ). Second, the upper internodes (internode 2-8 for Rio and internode 1-5 for Della) had higher sugar concentrations than the lower internodes in both genotypes. Third, total sugar concentrations were significantly increased at two stages in both genotypes: (i) the first 10 days after anthesis and (ii) from 22 to 32 DAF (Additional file 9). We also characterized the water content dynamics of both genotypes from 10 to 38 DAF (Fig. 2e; Additional file 9). Della and Rio had juicy stems and their internode water contents remained stably high $(\sim 70 \%$ to $80 \%)$ during stem sugar accumulation, except for an obvious decrease at 38 DAF in Della. Slight, but statistically significant, decreases in water contents were observed at $32 \mathrm{DAF}$ for most of the internodes in both genotypes, matching slightly increased Brix levels. Generally, the upper internodes had lower water contents compared to lower internodes (Fig. 2e).

\section{Comparative transcriptome analysis of sugar-accumulating stems}

Batch effects between the three RNA-seq data sets were removed (see "Methods", Additional files 4 and 10). The 


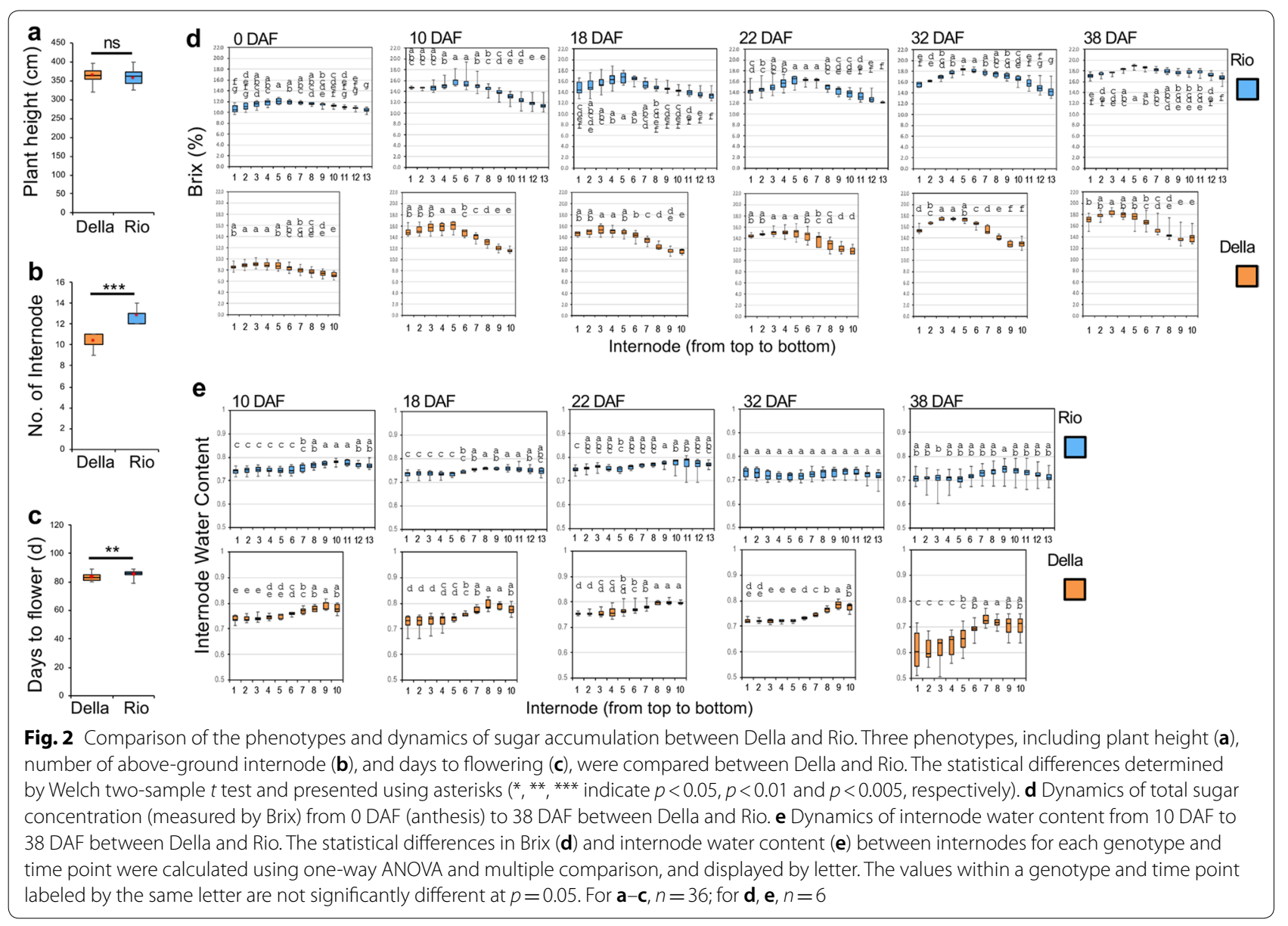

developmental timelines for the five genotypes were aligned relative to their anthesis (Fig. 1) [12, 26, 45, 77]. Principal component analysis (PCA) showed that $\sim 40 \%$ of transcriptome variances between samples were explained by PC 1 and 2, which appeared to be associated with developmental stages and genotypes, respectively (Fig. 3). Our previous study shows that the Rio-converted line R9188 is partially active in primary metabolism and has an intermediate stem sugar concentration. The PCA results showed that $\mathrm{R} 9188$ stem transcriptomes were differentiated from those of Rio and BTx406. Della stem transcriptomes fell between Rio and R9188, with SIL-05 stem transcriptomes grouped close to Della A11 and A25. Similarly, hierarchical clustering $(\mathrm{HC})$ results (Additional file 11) grouped the transcriptomes into five clusters and identified those enriched with sugar-accumulating internodes: (i) some developmental stages of Della, when soluble sugars are actively accumulated, were clustered with Rio stems (cluster 3); (ii) SIL-05 stem samples were clustered with R9188 stems (cluster 4). Overall, PCA and HC results suggest that metabolic active stem samples with high or intermediate sugars tend to group together.

\section{Cellulose synthetic genes}

Non-structural carbohydrates (sugars/starch) and structural carbohydrates (cell wall components) represent major carbon reserves in the stem during post-anthesis. Sugars and starch together account for $\sim 50 \%$ of stem dry weight, whereas structural carbohydrates account for $\sim 30 \%$ [26]. Representative sweet varieties had higher starch content (ranging from $\sim 3$ to $10 \%$ ) than grain sorghum lines $(<2 \%)$, supporting starch as an important carbon reserves in stem [11]. We compared the expression dynamics of primary metabolic genes to examine whether a similar expression trend could be observed in Della and SIL05 for the gene that was differentially expressed between Rio and BTx406/R9188 (Additional files 5 and 12). While considering the quantitative expression differences between genotypes, we primarily focused on the fold changes of gene expression within a genotype due to limitations in quantitative cross-comparison between data sets.

Several CesA genes were highly expressed in sweet sorghum genotypes, but significantly decreased in BTx406/ R9188 after flowering (Fig. 4a). These Ces $A$ genes belong 


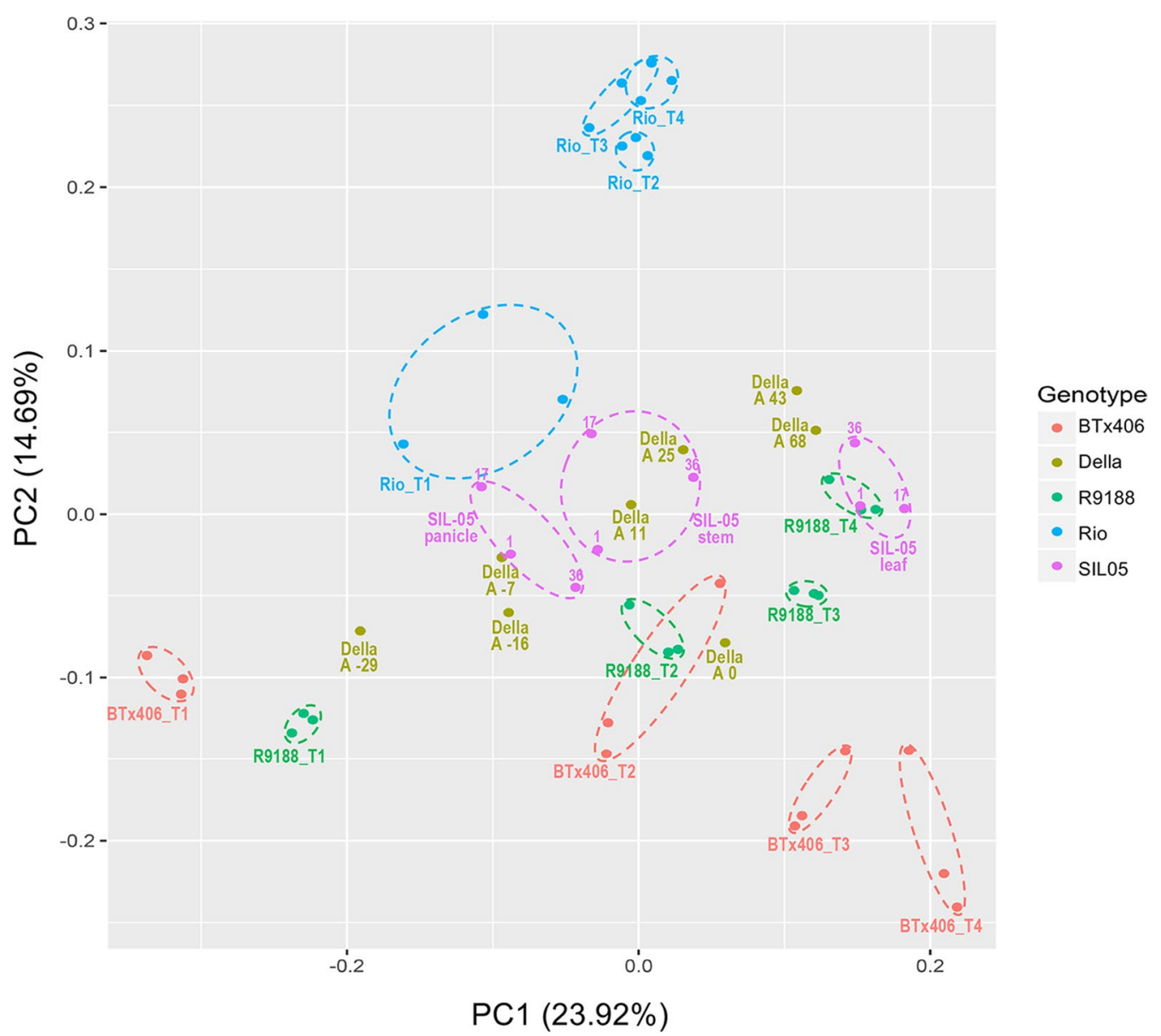

Fig. 3 PCA analysis. PC1 and PC2 variance of expression between RNA-seq samples and related developmental stages and genotypes, respectively, is shown

to the ancestral clusters CesA_AC4, CesA_AC5, and CesA_AC6 in phylogeny and are associated with PCW cellulose synthesis [63]. In contrast, the expression of CesA genes corresponding to SCW cellulose synthesis (Sobic.001G224300 and Sobic.002G205500) decreased post-anthesis in all genotypes. Several $C s l$ genes were differentially expressed between sweet and non-sweet genotypes. A CslF gene that has a major role in synthesis of mixed-linkage $(1,3 ; 1,4) \beta$-glucan (MLG) maintained its expression levels in the sweet genotypes with particularly high expression in Della and Rio, but decreased from pre-anthesis stages in non-sweet genotypes [73, 91]. Similarly, a CslA gene (Sobic.007G137400) decreased its expression in BTx406/R9188, but maintained a seemingly higher expression levels in sweet genotypes [92]. Its homologs in Arabidopsis are both responsible for mannan synthesis and affect cell wall integrity and organization [93]. Two highly expressed genes that encode homogalacturonan $\quad \alpha$-1,4-galacturonosyltransferase (GAUT) and are possibly responsible for pectin synthesis were significantly decreased in BTx406/R9188 after flowering, but were either stable or upregulated compared to anthesis stage in sweet sorghum [92]. Three xyloglucan galactosyltransferase (XGT) genes exhibited similar trends: their expression levels in BTx406/R9188 were significantly downregulated when comparing to the anthesis stage, but such downregulation was not observed after flowering in the sweet genotypes. More interestingly, two highly expressed genes encoding endo-1,4- $\beta$ glucanase (CAZy ID: GH9) were decreased in BTx406/ R9188, but remained high or upregulated expression in sweet genotypes after flowering. The Arabidopsis homolog (AT5G49720, KOR1) of the two GH9 genes can interact with CesA complex and is required for cellulose deposition [94-96], while the other Arabidopsis homolog (AT1G19940) affects cell wall crystallinity, secondary cell wall development, and biomass of Arabidopsis plants [96]. Overall, the genes highlighted here, CesA, Csl, XGT, $G A U T$, and $G H 9$, are important for the synthesis of cellulose, pectin, and hemicellulose (including xyloglucation, 


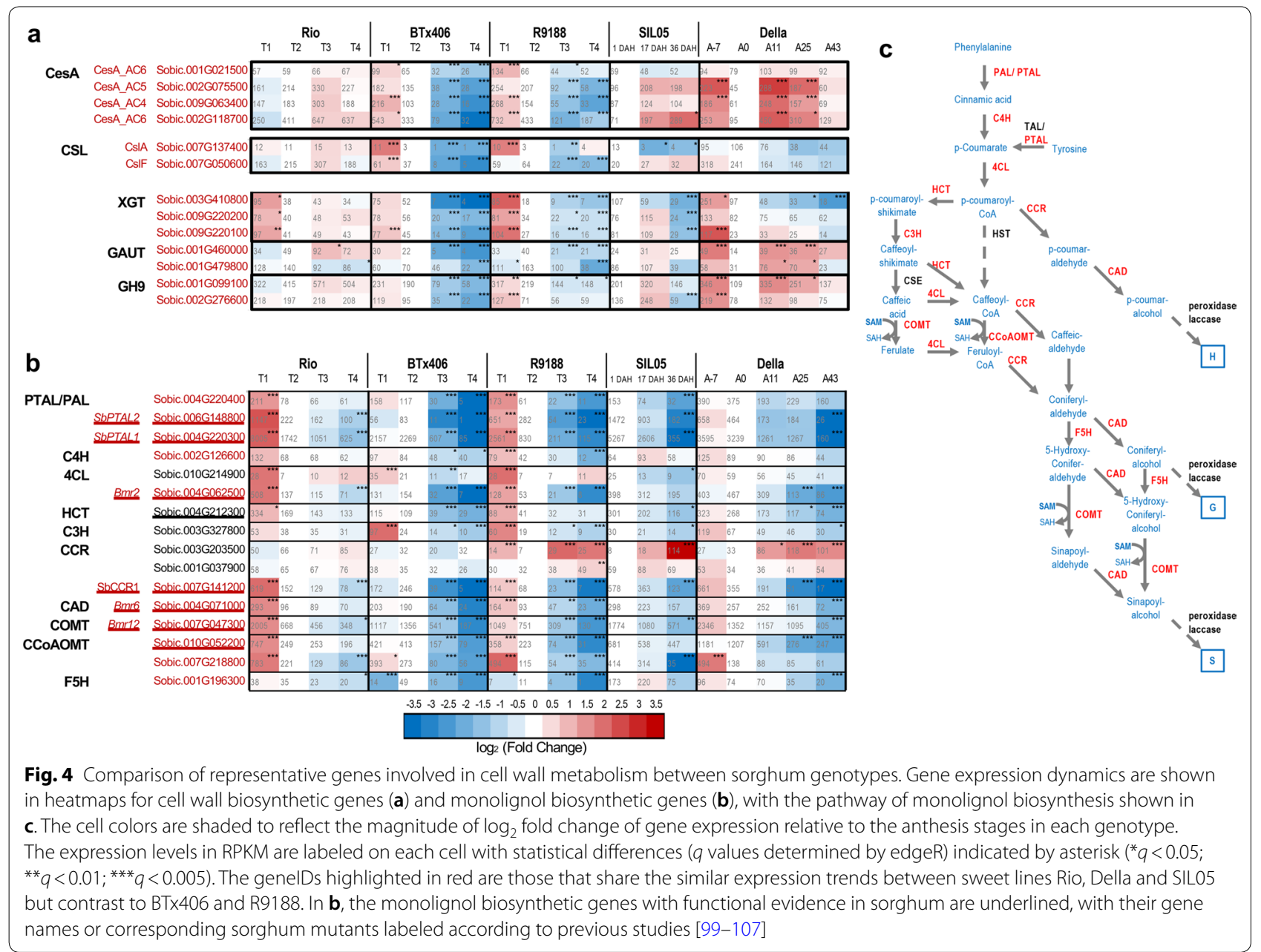

mannan, and MLG), which are the major components of primary cell wall [92]. Also, MLG is suggested as a storage form of stem glucose when comparing the carbon allocation differences between grain, sweet, and wild sorghum lines $[97,98]$. The expression analysis of cellulosic genes suggests that sweet sorghum stem tissue maintains an active primary cell wall development during postanthesis that could serve as a significant carbon demand.

\section{Monolignol biosynthetic genes}

Extensive functional and structural studies in sorghum have identified and characterized the major genes controlling key steps of monolignol biosynthesis. These genes include those encoding the first and the third enzymes in the phenylpropanoid pathway (PAL and 4CL, respectively) that impact on the metabolic flux of monolignol precursors $[99,100]$, and several downstream genes encoding the enzymes (HCT, CCR, CAD, COMT, and CCoAMOT) that alter overall lignification and/or monolignin ratios [101-107]. Here, the RNA-seq data sets confirmed that these major functional genes are among those with the highest expression levels in their own families (Fig. 4b, c). The monolignol pathway was active in all the genotypes before flowering and was gradually downregulated after stem maturation. Sweet genotypes had higher expression levels of these genes during postanthesis compared to BTx406/R9188, with statistical differences observed between Rio versus BTx406/R9188.

Previous studies on sorghum $P A L$ genes have identified two subgroups encoding the enzymes active for L-Phe deamination (PAL) and L-Phe/-Tyr deamination (PTAL), respectively [100]. Expression profiling showed that two SbPTALs (Sobic.004G220300 and Sobic.006G148800) and one SbPAL (Sobic.004G220400) had the highest expression levels in stem and their expression decreased remarkably during post-anthesis in BTx406/R9188. In contrast, the SbPTALs and SbPAL maintained their expression levels from $\sim 50$ to several hundreds of RPKM in sweet genotypes, significantly higher than those in BTx406/R9188 (Fig. 5a). Consistent with the higher expression of SbPTALs in sweet sorghum, L-tyrosine content was decreased in BTx406/R9188, but remained 


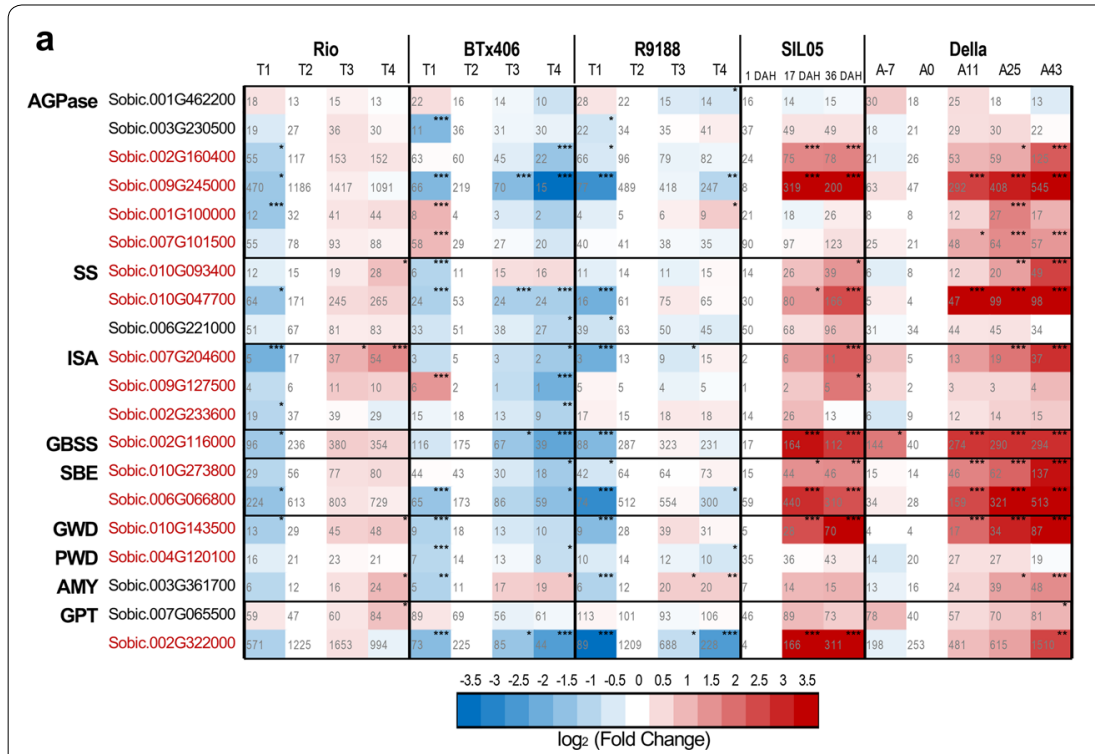

b

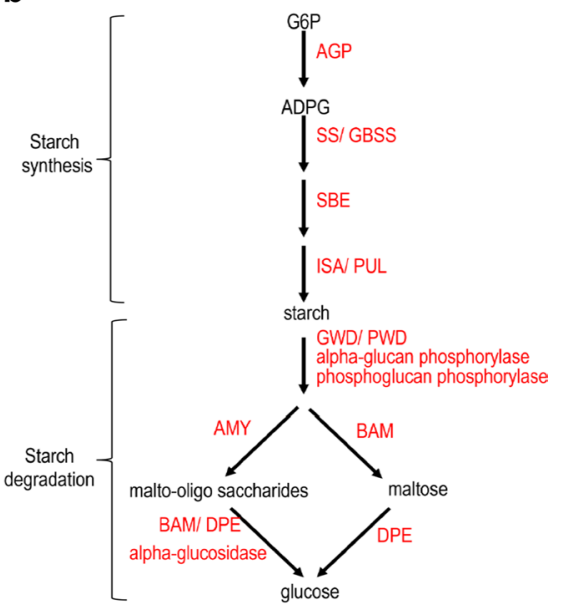

Fig. 5 Comparison of starch-metabolic genes between sorghum genotypes. The expression dynamics of representative starch-metabolic genes were compared between sorghum genotypes and shown in heatmap (a), with the starch-metabolic pathway shown in $\mathbf{b}$. The cell colors are shaded to reflect the magnitude of $\log _{2}$ fold change of gene expression relative to the anthesis stages in each genotype. The expression levels in RPKM are labeled on each cell with statistical differences ( $q$ values determined by edgeR) indicated by asterisk $\left({ }^{*} q<0.05 ;{ }^{* *} q<0.01 ;{ }^{* * *} q<0.005\right)$. The genelDs highlighted in red are those shared the upregulation expression trends between sweet lines Rio, Della and SIL05 but contrast to BTx406 and R9188

stable over time in Rio [12] (Additional files 4, 13), which could support the metabolic flux of phenylpropanoid biosynthesis in sweet sorghum. The other two enzymes that could influence the metabolic flux of precursors of flavonoids and lignins are $\mathrm{C} 4 \mathrm{H}$ and $4 \mathrm{CL}[99,100]$. Indeed, the most highly expressed $\mathrm{C} 4 \mathrm{H}$ gene (Sobic.002G126600) was significantly decreased in BTx406/R9188 compared to Rio at 15 DAF but was stable in the sweet genotypes. The predominant $4 C L$ gene (Sobic.004G062500, $B m r 2)$ exhibited an expression pattern similar to $C 4 H$ : its expression started to decrease in BTx406/R9188 at 10-days after flowering (T3), while in sweet genotypes, it remained stable until 15-25 days after flowering (for Rio and Della, respectively).

For the genes encoding downstream enzymes in the monolignol pathway, expression of several major functional genes including CCR, CAD, COMT, CCOAOMT, and $F 5 H$ was decreased from 10 DAF in BTx406/R9188, but such an expression trend was not detected until 15-25 DAF in sweet genotypes (Fig. 4b). Several of these predominantly expressed genes encode the functional enzymes for each family, of which the structural information and substrate kinetics had been experimentally validated previously, such as SbHCT [103], SbCCR1 [107], SbCAD2 [106], SbCOMT [105], and SbCCoAOMT [104]. Particularly, CCoAOMT catalyzes the methylation of caffeoyl-CoA to feruloyl-CoA, whereas COMT catalyzes the methylation of caffeic acid, 5-hydroxyconifer-aldehyde, or 5-hydroxyconifer-alcohol to facilitate $\mathrm{S}$ lignin production. Both COMT and CCoAOMT use $S$-adenosyl-L-methionine (SAM) as the methyl donor $[104,105]$. In the RNA-seq data set from Rio/BTx406/R9188, three SAM synthases (SAMS) showed significantly higher expression levels in Rio than those in BTx406/R9188, while several genes required for SAM metabolism were highly expressed [108] (Additional file 13). Similarly, using our previously published metabolome results from the same samples for RNA-seq dataset1, we showed that SAM content was stable in Rio over the stages, but were undetected after the T1 stage in BTx406/R9188, indicating that Rio could have higher levels of SAM compared to BTx406/R9188 [12] (Additional files 4, 13). Overall, most of the monolignol biosynthetic genes continuously decreased when compared to the pre-anthesis and anthesis stages in BTx406/R9188, but in sweet genotypes, they were relatively stable at early post-anthesis stages after an initial decrease from pre-anthesis stage. This suggests that active monolignol biosynthesis is maintained at early stages of post-anthesis in sweet sorghum.

\section{Starch biosynthetic genes}

Based on homologous and orthologous relationships between maize and sorghum genes, we identified genes encoding key enzymes in starch metabolism, including AGPase, SS, GBSS, SBE, ISA, GPT, GWD, PWD, and AMY (Additional file 12). We identified two AGPase small subunit genes and four AGPase large subunit genes, with Sobic.003G230500 and Sobic.007G101500 
encoding the predictively cytoplasm-localized small and large subunits, respectively (Fig. 5). Interestingly, several AGPase subunits predicted to have plastidial localization (Sobic.002G160400, Sobic.009G245000, and Sobic.001G100000) were significantly upregulated in the sweet genotypes during sugar accumulation, but their expression levels were stable or decreased in BTx406/ R9188. In contrast, the plastid-localized AGPase small subunits did not differ in expression trends between sorghum genotypes. Moreover, we identified two GPTs in sorghum (SbGPT1, Sobic.007G065500 and SbGPT2, Sobic.002G322000), of which the homologs in Arabidopsis function in G6P translocation into plastids and are responsible for providing G6P for the oxidative pentose phosphate pathway (OPPP) in specific tissues or fueling starch synthesis in non-green tissues, respectively $[109,110]$. Expression of SbGPT2 but not SbGPT1 was upregulated and remained at high levels in sweet genotypes, but dramatically decreased in BTx406/ R9188 (Fig. 5). Particularly, the Brix of introgression line R9188 can reach a high level comparable to Rio, but is not maintained and decreases at post-anthesis stages [12]. Among all the starch-related genes, SbGPT2 is the only gene whose expression dynamics correlates well with soluble sugar levels at all stages [12]. Both AGPase and GPT2 expression data indicated that ADP-glucose synthesis likely occurs in plastid and is highly active in sweet sorghum. Furthermore, several starch biosynthetic genes showed coordinated expression patterns like those observed in AGPase: (i) upregulation over the time course of stem sugar accumulation in sweet genotypes; (ii) significantly higher expression levels in Rio than in BTx406/R9188, and (iii) differential expression in Della and SIL05. They include two SS (Sobic.010G047700, Sobic.010G093400), one GBSS (Sobic.002G116000), two ISA (Sobic.007G204600, Sobic.009G127500), and two $S B E$ genes (Sobic. 010G273800, Sobic.006G066800). The co-expression patterns between these starch biosynthetic genes are consistent with the notion that starch biosynthetic enzymes from multiple pathways form complexes in maize endosperm amyloplasts [56]. In addition, SbGWD (Sobic.010G143500) and SbPWD (Sobic.004G120100) with key roles in starch degradation also showed upregulated expression in sweet genotypes but not in BTx406/R9188. Similarly, a model of starch metabolism in Della has been proposed based on the expression of starch-metabolic genes from the Della RNA-seq data sets, and the model supports the activation of starch metabolism as sugar accumulates in sweet sorghum stem [11]. Taken together, the activation of starch-metabolic genes is associated with stem sugar levels and sink strength: all three sweet genotypes maintained high and upregulated expression of starch genes; R9188 with intermediate stem sugar [12] had lowered expression levels in some starch genes compared to sweet genotypes, including those encoding $S b G P T 2$, AGPase (Sobic.001G100000, Sobic.007G101500), and SS (Sobic.010G047700, Sobic.010G093400).

\section{Sucrose-metabolic genes}

Sucrose levels could be influenced by three sets of enzymes that are directly involved in channeling sucrose into primary metabolism: (i) invertase (INV) hydrolyzes sucrose into glucose and fructose; (ii) sucrose synthase (SuSy) hydrolyzes sucrose into fructose and UDP glucose; (iii) sucrose-phosphate synthase (SPS) and sucrose-phosphate phosphohydrolase (SPP) resynthesize sucrose (pathway in Fig. 6). The INVs are grouped into alkaline-neutral INVs (INVANs) and acid INVs based on their optimum $\mathrm{pH}$, the latter being classified into cell wall INVs (INVCWs, insoluble) and vacuolar INVs (INVVRs, soluble). We identified $18 I N V s$ in sorghum [111], including seven INVANs, nine INVCWs, and two INVVRs, Additional file 14). We compared the $I N V \mathrm{~s}$ here with those identified in a sugarcane-sorghum comparative study [112] and found the $I N V$ s to be identical, with an additional INVCW (Sobic.001G099700) not expressed in the sorghum RNA-seq data sets. First, three INVCWs (Sobic.006G255600, Sobic.004G163800, and Sobic.003G440900) expressed in stems with varied expression patterns among genotypes, appeared not to be related to sugar accumulation (Fig. 6). Second, all seven INVANs were expressed. Their expression patterns varied among genotypes and time points, but were not directly correlated to sucrose accumulation, indicating tightly regulated sucrose metabolism in cytosol. Third, the INVVR (Sobic.004G004800) with the highest expression levels among all $I N V \mathrm{~s}$ was sharply decreased at anthesis in all genotypes, a prerequisite for sugar accumulation in vacuole. Three SuSy genes were highly and differentially expressed: Sobic.001G344500 with the highest expression level was remarkably downregulated in all the genotypes, correlating with decrease of sucrose cleavage activity in Rio during sugar accumulation [113]; expression dynamics of Sobic.010G072300 and Sobic.001G378300 differed between genotypes. Several highly expressed SPSs and SPP (Sobic.004G151800) were stably detected and no clear trends were observed between sweet versus non-sweet genotypes. Furthermore, three Trehalose 6-Phosphate Synthase (TPS) and two Trehalose Phosphate Phosphatase (TPP) genes were highly transcribed. TPS and TPP genes jointly control the biosynthesis of trehalose 6-phosphate (T6P), an important signal and negative feedback regulator of sucrose levels [114]. One TPP gene (Sobic.002G303900) showed distinct expression patterns in sweet versus non-sweet sorghum. It was 


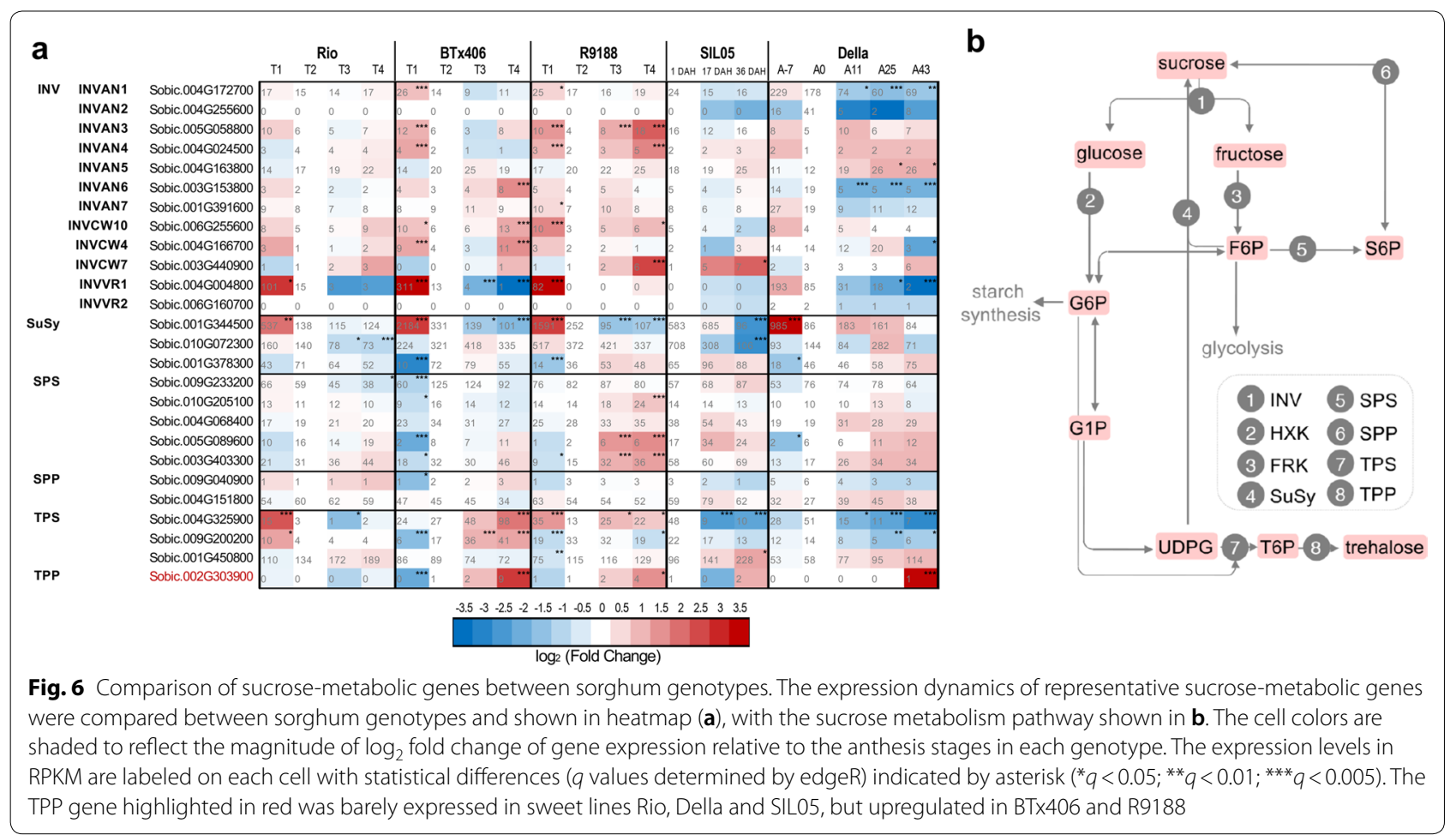

barely expressed in sweet genotypes but upregulated in Btx406/R9188 [12]. Functional studies are needed to elucidate their potential roles in carbon metabolism. It is worth noting that none of these sucrose-metabolic genes, except for TPP, showed upregulation when comparing sweet versus non-sweet genotypes, unlike those observed in cell wall and starch-metabolic genes (Figs. 4, 5,6 ), suggesting two possibilities: sucrose metabolism is not a major limiting factor for stem sugar accumulation, or the regulatory role of sucrose-metabolic pathway lies in post-transcriptional levels. Taken together, the expression of SPSs, SPPs, SuSys and seven intracellular INVANs suggest active sucrose metabolism in stems, supporting previous conclusion that sucrose may be inverted and resynthesized, while a significant portion was not metabolized during accumulation [40, 41].

\section{Sucrose transporters}

To identify candidate sucrose transporters responsible for stem sugar accumulation, we analyzed three families: SUTs, TSTs, and SWEETs. A comprehensive phylogenetic analysis of SWEETs from all the sequenced species within Angiosperms defines four clades [47]. Results of SWEETs substrate selectivity have been reported in several species, such as Arabidopsis [48-50], rice [54], maize [52, 53], cucumber [51], and other species [115]. Published data suggest that the SWEET substrate selectivity correlates well with its phylogenetic clade (Additional file 15), indicating that only clade III SWEETs are likely to transport sucrose. However, the physiological functions of SWEETs remain to be characterized individually, which could be determined by their spatio-temporal expression patterns and/or the metabolic pathway that SWEETmediated transport process could affect. According to our bioinformatics search, we identified 23 SWEETs and validated them to group into the four previously defined clades (Additional file 16) [47], with the designation of 20 SbSWEETs being consistent with the previous report [46]. By contrast, Mizuno et al. [45] identified 23 SWEETs including Sobic.003G149000, Sobic.003G038700, and Sobic.003G038800. Sobic.003G149000 contains two complete MtN3 domains and is designated SbSWEET17 (Additional files 6, 7, 16). Sobic.003G038700 is grouped with clade II SWEETs and has two complete MtN3 domains, but Sobic.003G038800 has not, which is probably a tandemly duplicated copy of Sobic.003G038700 (Additional files 6, 7). These two putative SWEETs specifically expressed in the inflorescence at relatively lower levels compared to other SWEETs in the expression database MOROKOSHI (Additional file 7) [45, 84]. We focused on clade III SWEETs that could transport sucrose. Among the six differentially expressed clade III SbSWEETs, SbSWEET13A (Sobic.008094000) exhibited highest expression level in stems and was upregulated in all the genotypes (Fig. 7) [45]. Previously, SbSWEET13A was not found to be differentially expressed when 


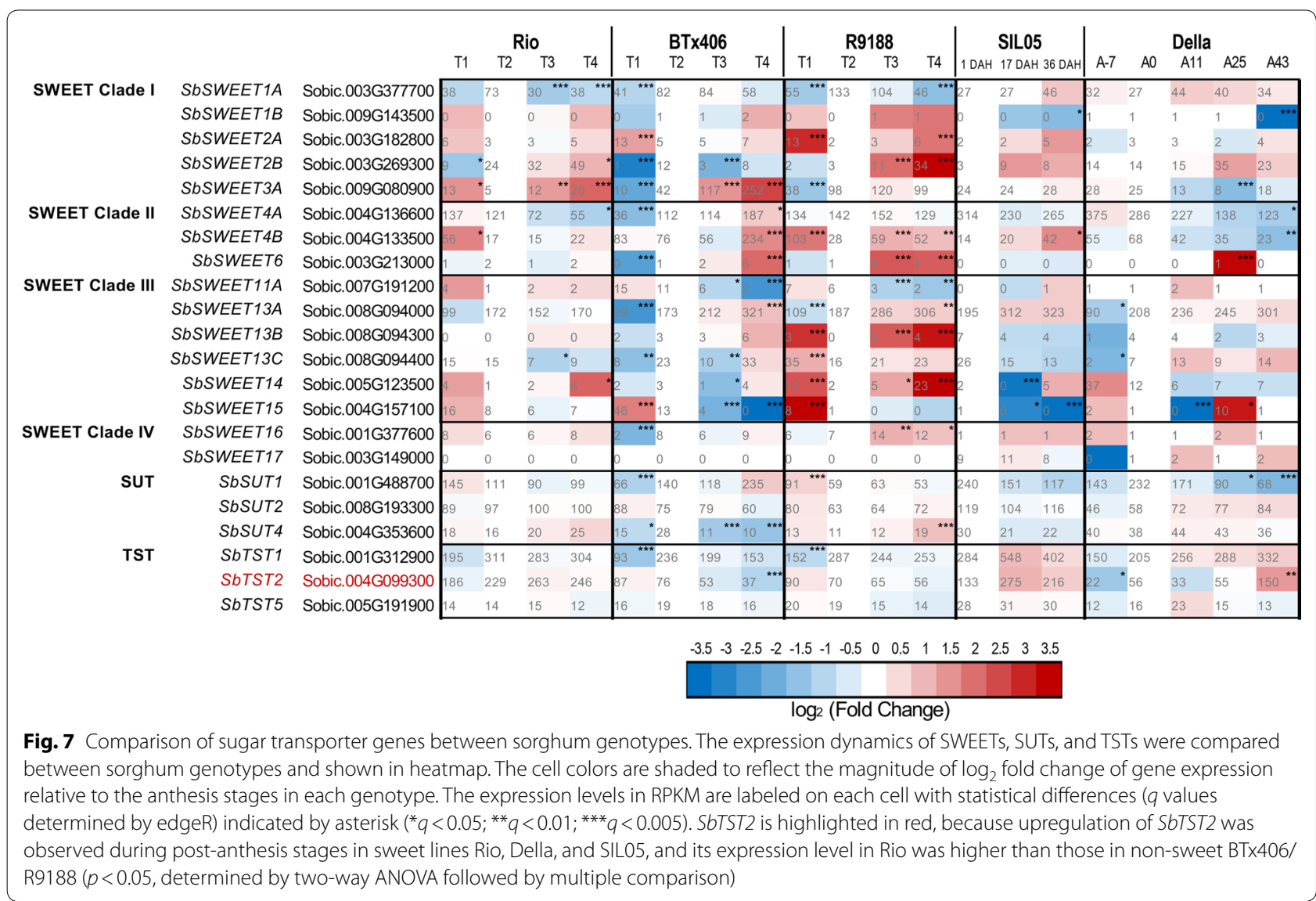

compared between two grain and sweet sorghum lines [46]. Here, integration of several data sets demonstrated that SbSWEET13A was upregulated in stem tissues from all the investigated genotypes, probably excluding its role in determining sugar accumulation difference between grain and sweet sorghum (Fig. 7). Interestingly, the tandem-duplicated SbSWEET13A/B/C showed different expression levels and spatio-temporal specific patterns according to our data and other databases (Fig. 7). $S b S W E E T 13 A / B$ were preferentially expressed in green tissues, while $S b S W E E T 13 C$ was also expressed in roots, suggesting their functional divergence. SbSWEET11A exhibited lower expression levels in sweet genotypes than in the non-sweet genotypes, although its expression level was relatively lower than other clade III SWEETs. It is possible that SbSWEET11A might be highly expressed at particular cell types in stem tissue, leading to its expression level being diluted when measured in whole tissues. In addition, SbSWEET13A and SbSWEET11A appeared to be positively and negatively correlated to internode maturity or stages in non-sweet genotypes, but such a correlation was not observed in sweet genotypes, the biological significance of which needs further functional studies. The other clade III SbSWEET genes failed to generate a clear expression trends between sweet and non-sweet genotypes (Fig. 7), suggesting that SWEETmediated sucrose transport may not be a major difference between sweet and non-sweet genotypes. Mizuno et al. [45] proposed that SbSWEET13A and 3A are involved in sucrose efflux from leaf; however, SbSWEET3A was grouped into clade I and homologous to other clade I SWEET3s from rice and maize (Fig. 7, Additional file 6) [52-54]. Similarly, the SbSWEET13A homolog in sugarcane (SsSWEET13C) was highly expressed in leaf mature zone and internode sclerenchyma cells, but expressed at very low levels in stem parenchyma, supporting a proposed role of SWEET13 in sucrose efflux in leaves [116]. SbSWEET4A, 4B, and 4C were suggested as candidates for sucrose transportation in panicle and stem, respectively, due to their spatial expression preference [45]. Here, SbSWEET4A/B/C are grouped into clade II together with their maize orthologs, consistent with the previous SWEET phylogenetic analysis [47]. Thus, the proposed roles of SbSWEET4s would be questioned because of their phylogeny-function correlation. It should be noted that the SWEET phylogenetic tree here resembles the one that covered all the sequenced species of Angiosperms, but differs from the tree reported 
in sorghum $[45,47]$. This discrepancy may be due to the following differences in phylogenetic analysis: (i) species used for analysis; (ii) methods for amino acid sequence alignment; (iii) parameters for constructing phylogenetic tree.

Additionally, we identified three SUTs that were differentially expressed in at least one genotype (Fig. 7). Among the two highly expressed SUTs (SbSUT1, Sobic.001G488700; SbSUT2, Sobic.008G193300), only SbSUT2 had a slightly higher expression level in sweet genotypes compared to BTx406/R9188 during post-anthesis, suggesting it as a candidate transporter related to stem sugar accumulation. The expression profiles of SbTSTs showed that Sobic.001G312900 and Sobic.004G099300 also had higher expression levels (Fig. 7); they are homologous to the TST1 and TST2 in Arabidopsis [117] and sugar beet [118]. TST2 is the only TST member that can transport sucrose and is related to sucrose storage in vacuoles, which is confirmed in several species, including sugar beet [118], melon [119], and watermelon [120]. Here, SbTST2 was highly expressed in sweet genotypes Rio and SIL-05, but had lower expression in BTx406/R9188 (two-way ANOVA, $p<0.05$ ) and downregulation in BTx406 (Fig. 7). In Della, SbTST2 also showed a gradual increase in expression levels, particularly at A43 and A67 stages (Fig. 7; Additional file 12). The discrepancy between SbTST2 expression and sugar accumulation in Della could suggest post-translational regulation of its activity [121]. Overall, comparative expression analysis of sucrose transporters highlights the candidates that are likely be involved in sucrose transport and accumulation in sorghum stems, showing that SbTST2, but not SWEETs, is the top candidate for functional study and genetic improvement $[42,46]$. Further investigations are needed to elucidate the physiological roles of these transporters in sorghum.

\section{Discussion}

\section{Common metabolic network contributing to carbon sink} strength in sweet sorghum internodes

The purposes of this study were to address whether candidate pathways/genes are specific for a given sweet sorghum genotype and to identify common expression features of primary metabolism and sugar transportation between different sweet sorghum genotypes. We, therefore, compared the dynamic transcriptomes of sugaraccumulating internodes between three unrelated sweet sorghum genotypes, with an emphasis on genes functioning in carbon utilization and sugar transport.

Previous studies have focused on sugar transporters SUTs, SWEETs, and TSTs that may contribute to stem sugar accumulation in sorghum. Analysis of SUTs expression in several tissues from grain sorghum BTx623 and sweet sorghum Rio at vegetative and anthesis stages showed variation of SUT5 and SUT6 but not of the highly expressed SUT1 and SUT2 [44]. Bihmidine et al. [42] compared SUT expression between grain and sweet sorghum and concluded that SUTs are unlikely to account for stem sugar in sorghum, because they showed differential expression in leaf tissue but not in stem tissue. The results of SUT expression in SIL05 revealed that their expression varied spatio-temporally but not more than twofold in the stem [45]. Our group characterized transcriptome dynamics over the period of sugar accumulation and the SUTS' results are consistent with the previous studies: (1) SUT1, 2 and 4 are highly expressed, while variation of expression exists between members (SUT1 > SUT2 > SUT4 at expression levels; Fig. 7); (2) variety-specific difference in expression levels were only observed for SUT1 and SUT2 at certain time points in the stem; (3) generally, the expression dynamics over time points and their comparison between several studied genotypes do not support roles in determining stem sugar difference between sorghum types. It is worth to note that the very low-expression levels of SUT5 and 6 could deny their biological significance as transporters, and also makes it hard to ascertain differential expression due to high variance. Still, we could not exclude the possibility that SUTS and SUT6 may be highly expressed at particular cell types in stem, similar to the above-mentioned case for SbSWEET11A. More recently, the expression analysis of SbTSTs suggests SbTST2 as a candidate gene accounting for stem sugar difference due to its differential expression between a grain and a sweet sorghum line [46]. Five TSTs were identified based on our annotation [12]. The integrated expression data here show that TST1 and TST2 are the top two highly expressed TSTs followed by TST5. Similar to the previous study, we only observed differential expression for TST2 between grain and sweet genotypes with TST2 upregulated in all sweet genotypes (Fig. 7) [46]. Taken together, SbSUT2 and SbTST2, but not clade III SWEETs, may be candidate transporters for sucrose transportation in the stem, and SbTST2 could be directly responsible for sucrose transportation into storage vacuoles $[42,46]$.

Though a candidate gene directing vacuolar storage of sucrose has been proposed [46], important biological questions remain to be addressed, such as: what factors determine that more sucrose is available in sweet sorghum than in grain sorghum for vacuolar storage? To what extent does carbon metabolism contribute to sugar accumulation? To address these questions, a system and integrated approach is needed to provide a cohesive and comparative picture of stem carbohydrate metabolism between distinct types of sorghum. Previous studies indicated that sucrose in vacuoles, starch in plastids, 
and carbohydrates in cell walls constitute the three major stem carbon reserves of sweet sorghum [11, 26]. Unlike the previous RNA-seq studies using one genotype, the major novelty of this comparative analysis is that we sought to replicate the comparison between sweet versus non-sweet genotypes using the RNA-seq data sets from Della and SIL05 [12, 26, 45]. Thus, such a comparison identified the common expression features in several pathways of primary metabolism and sugar transportation that differed from those seen in non-sweet BTx406/ R9188. They are: (i) genes for cellulose, pectin, and hemicellulose synthesis were upregulated or highly expressed in sweet genotypes, but decreased in non-sweet lines; (ii) genes for phenylpropanoid and monolignol synthesis were decreased slower in sweet genotypes than in non-sweet lines; (iii) genes for starch metabolism were upregulated in sweet genotypes; (iv) when multiple sweet sorghum genotypes were investigated, the previous indications of INV or SWEETs as candidate genes for stem sugar accumulation were not strongly supported $[26,45]$. Our comparison supports SbTST2 as a top candidate for functional studies and also identifies TPP as a candidate gene. While the transcriptional dynamics of cell wall and starch biosynthesis has been reported in Della, the association between cell wall/starch biosynthesis and stem sugar accumulation is established in the current study through comparative RNA-seq analysis. Such an association suggests that carbon allocation in stem might be coordinated among the carbon metabolic pathways (see below). In addition, the expression of 16 candidate genes highlighted in our analysis was determined by qRT-PCR. The RNA-seq and qPCR expression patterns of these 16 genes in Rio, BTx406, and R9188 were highly similar (Additional file 17).

The manners in which these metabolic pathways contribute to carbon utilization are different. Monolignol pathways are generally decreased from pre-anthesis stage, but the extent or rate of such decrease is less in sweet genotypes than in the non-sweet (Fig. 5). Expression of several key genes involved in primary cell wall components (cellulose, pectin and MLG) remained stable at early post-anthesis stages in sweet sorghum, but was gradually reduced in non-sweet genotypes (Fig. 4). By contrast, expression levels of many starch synthetic genes were well correlated with stem sugar accumulation, SbGPT2 appearing to be a limiting factor for starch due to its potential role of providing G6P (Fig. 7). Close examination of these metabolic pathways highlights several candidate genes (e.g., AGPase, GPT2, and CesA) that may be used for regulating stem sugar accumulation through up- or downregulation of specific pathways. Additionally, we examined the $D$ gene expression (Sobic.006G147400), because it is a major regulator of stem juiciness and aerenchyma formation [30-33]. Results showed that the $D$ gene determined stem juiciness, but did not affect the sugar concentration in juice [31]. Indeed, the $D$ gene was not expressed in Rio, R9188, Della, and SIL05, with very low-expression detected in BTx406 (RPKM 2; Additional file 12), consistent with the previous results that a nonfunctional $D$ gene is required for juicy stem, the prerequisite of sweet sorghum [30-33].

Although more research will be needed for further functional studies, the results allow us to propose a model for carbon allocation in sink stems of sweet sorghum (Additional file 18). In this model, sucrose transported from long distance could flow into four metabolic fates in storage parenchyma cells: first, sucrose inversion and re-synthesis for consuming energy could provide materials for starch and cell wall synthesis (glucose6-phosphate and UDPG, respectively); second, vacuolar storage of sucrose could serve not only as a carbon reserve, but also as a temporary pool for starch and cell wall metabolism, when sucrose supply fluctuates; third, starch synthesis and storage in plastids could flow from degradative products of sucrose; fourth, carbohydrates could be used for cellulose synthesis for primary cell wall and maintaining relatively active monolignol biosynthesis compared to grain sorghum. This carbon sink model represents carbon allocation in several new ways compared with the previous studies. (1) It provides transcriptomics evidence supporting the notion that carbon allocation within sorghum sink stem is likely to be coordinated by different carbon utilization routes and this is likely a common feature of the sweet sorghum stems. (2) It highlights and summarizes reliable candidate genes for modulating stem carbon compositions from previous identified pathways. (3) It provides transcriptomics evidence indicating that sucrose cleavage and re-synthesis could occur in sorghum stem. (4) It clarifies the potential roles of SWEETs in leaf sucrose efflux but not in the stem sugar difference between the sweet versus non-sweet genotypes.

The comparative transcriptome approach used herein has three advantages. (i) Integration of the three data sets: to overcome the limitations in differential expression analysis for data sets 2 and 3, the biological variance from similar tissues and time points (dataset1) was employed to identify differentially expressed genes in Della and SIL05 ("Methods"; Additional file 4); besides, differential expression analysis within each dataset allowed the association between expression dynamics with sugar accumulation but avoided potential problems raised from direct comparison of the genotypes between datasets, such as removal of the batch effects between datasets, intrinsic expression difference between genotypes unrelated to sugar. (ii) We consider genes involved in the three major carbohydrate reserves that are supported by previous 
phenotype results $[11,12,26]$ and, therefore, provide the metabolism-related transcriptome dynamics at genomewide level. (iii) The proposed model based on our comparative analyses not only provides promising candidate genes for bioenergy improvement, but also may serve as a guidance for understanding and manipulating carbon composition of sorghum stem at transcriptional level. In line with the notion of coordination between carbon utilization routes, this model suggests that it is possible to modulate stem biomass composition through up- or downregulation of specific primary metabolic pathways. Similar coordination between sucrose and starch metabolism has been well demonstrated and applied in the case of various sweet corns, which are caused by mutations in starch biosynthetic genes [122]. Generally, several mutants in starch defects result in increased sucrose content in maize kernels, representing the re-distribution of carbon from starch synthesis to sugar metabolism. Applying a similar concept in sorghum, stem starch biosynthesis might be knocked down to enhance sucrose accumulation in vacuoles via repression of GPT2 or key starch biosynthetic enzymes. Sucrose storage in vacuoles might be also enhanced by overexpressing SbTST2 [46]. Also, it might be possible to regulate the primary metabolic pathways downstream of T6P signal by modifying T6P content via transgenic SbTPPs. A similar approach of heterologous expression of TPP has been shown to be efficient in altering metabolism in maize endosperm [123, 124]. Additionally, stem biomass composition results from the sorghum mutants in monolignol biosynthetic could also be explained this way. Near isogenic lines (NIL) carrying the bmr6 mutation in grain sorghum background showed significantly increased total free soluble sugars, whereas the effect of bmr12 mutant varied depending on genetic backgrounds [125]. Decreased lignin contents are associated with slightly, though statistically not significant, increase in stem sugar concentration, [126].

Still, further improvement of the current model will be required. Particularly, functional validation of the candidate genes in sorghum is necessary to improve our understanding of the metabolic consequences of carbon utilization. On the other hand, incorporation of metabolomics and proteomics data in the future will refine the model, as transcriptomics data themselves have limitations in interpreting metabolism due to multiple layers of regulation at post-transcriptional, protein and metabolite levels $[127,128]$. Also, our interpretations may come with caveats based on the possibility that the expression data analyzed may not be truly representative in some instances, since some of the data sets are not replicated. Moreover, several cell wall components, such as xylan and glucan, account for considerable fractions of carbon utilization [26] are not included in the present model due to missing information on their metabolic genes in sorghum and closely related species.

\section{Conclusions}

Here, we have presented the first comparative transcriptome analysis of sugar-accumulating internodes in sorghum that is relevant to bioenergy research at a gene discovery level. The common transcriptome features indicate differences in several primary metabolic pathways between the sweet and non-sweet sorghums, suggesting the metabolic networks possibly coordinating carbon allocation and sink strength in the sorghum internode. Specifically, several genes, including those involved in cellulose and monolignol synthesis (CesA, $P T A L$, and CCR), starch metabolism (AGPase, SS, SBE and G6P-translocator $S b G P T 2$ ), and sucrose metabolism and transportation (TPP and TST2), were strongly correlated with the three sweet sorghum genotypes compared to the non-sweet lines, serving as candidates for functional studies of carbon manipulation in sorghum stem. This study also shows that a combination of multiple advanced resources (including metabolites, expression data sets, genotypes, and conditions of sorghum stem sink) provides a comprehensive and cohesive picture of the complexity of carbon sink strength in sorghum stem, which might not be achieved by a single data set. The many candidate genes identified here could be manipulated and studied to further our understanding and utilization of carbon allocation and/or sugar accumulation in bioenergy crops.

\section{Supplementary information}

Supplementary information accompanies this paper at https://doi. org/10.1186/s13068-019-1612-7.

Additional file 1. Pedigrees of sweet sorghum Rio, Della and SIL-05.

Additional file 2. Information of the RNA-seq samples used in this study.

Additional file 3. Variation in expression of the reference genes in sorghum before and after removal of batch effect.

Additional file 4. Supplementary methods.

Additional file 5. Annotation of genes involved in the primary metabolic pathways.

Additional file 6. Annotation and nomenclature of SbSWEETs.

Additional file 7. Spatio-temporal expression patterns of sorghum SWEETS.

Additional file 8. Primers used for real-time quantitative PCR (qPCR).

Additional file 9. Dynamics of internode Brix (a) and water concentration (b) over stem sugar accumulation.

Additional file 10. Distribution of the expression levels for all the RNA-seq data sets used before (a) and after (b) batch-effect removal. 
Additional file 11. Hierarchical clustering $(\mathrm{HC})$ of the RNA-seq data sets used.

Additional file 12. Selected differentially expressed genes in the cellulose biosynthesis, monolignol biosynthesis and starch and sucrose metabolic pathways.

Additional file 13. The dynamics of tyrosine and SAM and the expression levels of genes involved in SAM metabolism.

Additional file 14. Annotation of sorghum invertases (INVs) and their homologs and orthologs in maize.

Additional file 15. Summary of the substrate selectivity and phylogenetic clades of SWEET proteins.

Additional file 16. The phylogenetic tree of SWEET proteins from rice, maize and sorghum.

Additional file 17. Validation of RNA-seq results by qPCR.

Additional file 18. A proposed model for carbon partitioning and sink strength in sorghum internodes.

\section{Abbreviations}

ADPG: ADP-glucose; AGPase: ADP-glucose pyrophosphorylase; BAM: $\beta$-amylase; bmr: brown midrib; $\mathrm{C} 3 \mathrm{H}$ : 4-coumarate 3-hydroxylase; $\mathrm{C} 4 \mathrm{H}$ : cinnamate 4-hydroxylase; CAD: cinnamyl alcohol dehydrogenase; CCOAOMT: caffeoyl-coenzyme A 3-O-methyltransferase; COMT: caffeic acid O-methyltransferase; CCR: cinnamyl-CoA reductase; CesA: cellulose synthase; Csl: cellulose synthase-like; DAF: days to flowering; DAH: days to heading; DBE: starch debranching enzyme; DEG: differentially expressed gene; DPE: disproportionating enzyme; F5H: ferulate 5-hydroxylase; G1P: glucose-1-phosphate; G6P: glucose-6-phosphate; GBSS: granule-bound starch synthase; GPT: glucose-6-phosphate translocator; GWAS: genome-wide association analysis; GWD: glucan-water dikinase; HC: hierarchical clustering; HCT: hydroxycinnamoyltransferase; INV: invertase; INVAN: alkaline-neutral invertase; INVCW: cell wall invertase; INVVR: vacuolar invertase; ISA: isoamylase; PAL: phenylalanine ammonia-lyase; PCA: principle component analysis; PCW: primary cell wall; PGM: phosphoglucomutase; PHT: plant height; PTAL: L-Phe/-Tyr L-Phe/-Tyr ammonia-lyase; PWD: phosphor-glucan-water dikinase; QTL: quantitative trait loci; RPKM: reads per kilobase of exon per million mapped sequence reads; SAM: S-adenosyl-L-methionine; SBE: starch branching enzyme; SCW: secondary cell wall; SPP: sucrose-phosphate phosphohydrolase; SPS: sucrosephosphate synthase; SS: soluble starch synthase; SuSy: sucrose synthase; SUT: sucrose transporter; SWEET: Sugars Will Eventually be Exported Transporters; T6P: trehalose-6-phosphate; TPP: trehalose-6-phosphate phosphatase; TPS: trehalose-6-phosphate synthase; TST: Tonoplast Sugar Transporter; USDANPGS: United States Department of Agriculture National Plant Germplasm System; 4CL: 4-coumarate:CoA ligase.

\section{Acknowledgements}

This work is dedicated to the memory of Dr. Joachim Messing (1946-2019), who is a pioneer in molecular genetics, genomics and biotechnology, and made major contributions in the sequencing of rice, maize, and sorghum. This work was supported by the Selman Waksman Chair in Molecular Genetics (J.M.). We thank Dr. Dibyendu Kumar and the Waksman Genomic core facility for RNA-seq, Josh Gager and Rushabh Mehta for field assistance, and Paul Fourounjian for his revision of the manuscript. We thank Dr. Hugo Dooner for his critical edits and proof reading of the manuscript.

\section{Authors' contributions}

$Y L, W W$, and $J M$ conceived and designed the study. YL contributed to the fielding work. YL, WW, MT, and YF contributed to data analysis and interpretation. YL and JM drafted the manuscript, and all authors critically revised the final version of the manuscript. All authors read and approved the final manuscript.

\section{Funding}

This work was supported by the Selman Waksman Chair in Molecular Genetics (J.M.).

\section{Availability of data and materials}

All data generated or analyzed during this study are included in this published article and its additional information files.

\section{Ethics approval and consent to participate}

Not applicable.

\section{Consent for publication}

Not applicable.

\section{Competing interests}

The authors declare that they have no competing interests.

\section{Author details}

${ }^{1}$ Waksman Institute of Microbiology, Rutgers, The State University of New Jersey, Piscataway, NJ 08854, USA. ${ }^{2}$ School of Agriculture and Biology, Shanghai Jiao Tong University, 800 Dong Chuan Road, Shanghai 200240, China.

Received: 21 May 2019 Accepted: 9 November 2019

Published online: 20 November 2019

\section{References}

1. Lemonie R, Camera S, Atanassova R, Dedaldechamp F, AllaRio T, Pourtau $\mathrm{N}$, Bonnemain J, et al. Source-to-sink transport of sugar and regulation by environmental factors. Front Plant Sci. 2013;4:272.

2. Wardlaw IE. The control of carbon partitioning in plants. New Phytol. 1990;116:341-81.

3. Paul $M$, Foyer $C$. Sink regulation of photosynthesis. J Exp Bot. 2001:52(360):1383-400.

4. Ho L. Metabolism and compartmentation of imported sugars in sink organs in relation to sink strength. Annu Rev Plant Physiol Plant Mol Biol. 1988;39:355-78.

5. OsoRio S, Ruan YL, Fernie A. An update on source-to-sink carbon partitioning in tomato. Front Plant Sci. 2014;5:516.

6. Kebron TH, McKinley B, Mullet JE. Dynamics of gene expression during development and expansion of vegetative stem internodes of bioenergy sorghum. Biotechnol Biofuels. 2017;10:159.

7. Calvino M, Messing J. Sweet sorghum as a model system for bioenergy crops. Curr Opin Biotechnol. 2012;23(3):323-9.

8. Mathur S, Umakanth AV, Tonapi VA, Sharma R, Sharma MK. Sweet sorghum as biofuel feedstock: recent advances and available resources. Biotechnol Biofuels. 2017;10:146.

9. Wang J, Nayak S, Koch K, Ming R. Carbon partitioning in sugarcane (SaCcharum species). Front Plant Sci. 2013:4:201.

10. Zhang J, Zhang X, Tang H, Zhang Q, Hua X, Ma X, Zhu F, Jones T, Zhu X, Bowers J, et al. Allele-defined genome of the autopolyploid sugarcane Saccharum spontaneum L. Nat Genet. 2018;50(11):1565-73.

11. McKinley B, Casto A, Rooney W, Mullet J. Developmental dynamics of stem starch accumulation in Sorghum bicolor. Plant Direct. 2018;2:1-15.

12. Li Y, Wang W, Feng Y, Tu M, Wittich PE, Bate NJ, Messing J. Transcriptome and metabolome reveal distinct carbon allocation patterns during internode sugar accumulation in different sorghum genotypes. Plant Biotechnol J. 2019;17(2):472-87.

13. Paterson AH, Bowers JE, Bruggmann R, Dubchak I, Grimwood H, Haberer $G$, Hellsten U, et al. The Sorghum bicolor genome and the diversification of grasses. Nature. 2009;457(7229):551-6.

14. Deschamps S, Zhang Y, Llaca V, Ye L, Sanyal A, King M, May G, Lin H. A chromosome-scale assembly of the sorghum genome using nanopore sequencing and optical mapping. Nat Commun. 2018;9:4844.

15. McCormick RF, Truong FK, Sreedasyam A, Jenkins J, Shu S, Sims D, Kennedy M, Amirebrahimi M, Weers BD, McKinley B, et al. The Sorghum bicolor reference genome: improved assembly, gene annotations, a transcriptome atlas, and signatures of genome organization. Plant J. 2018;93(2):338-54.

16. Guo H, Jiao $Y$, Tan $X$, Wang $X$, Huang $X$, Jin H, Paterson AH. Gene duplication and genetic innovation in cereal genomes. Genome Res. 2019. https://doi.org/10.1101/gr.237511.118. 
17. Mullet J, Morishige D, McCormick R, Truong S, Hilley J, McKinley B, Anderson R, et al. Energy sorghum - a genetic model for the design of C4 grass bioenergy crops. J Exp Bot. 2014;65(13):3479-89.

18. Johnson SM, Cummins I, Lim FL, Slabas AR, Knight MR. Transcriptomic analysis comparing stay-green and senescent Sorghum bicolor lines identifies a role for proline biosynthesis in the stay-green trait. J Exp Bot. 2015;66(22):7061-73.

19. Boyles RE, Brenton ZW, Kresovich S. Genetic and genomic resources of sorghum to connect genotype with phenotype in contrasting environments. Plant J. 2019;97(1):19-39.

20. Xin Z, Wang M, Barkley NA, Burow G, Franks C, Pederson G, Burke J. Applying genotyping (TILLING) and phenotyping analyses to elucidate gene function in a chemically induced sorghum mutant population. BMC Plant Biol. 2008;8:103.

21. Jiao Y, Burke J, Chopra R, Burow G, Chen J, Wang B, Hayes C, Emendack $Y$, Ware D, Xin Z. A sorghum mutant resource as an efficient platform for gene discovery in grasses. Plant Cell. 2016;28(7):1551-62.

22. Addo-Quaye C, Buescher E, Best N, Chaikam V, Baxter I, Dilkes B. Forward genetics by sequencing EMS variation-induced inbred lines. G3 (Bethesda). 2017;7(2):413-25.

23. Lowe K, Wu E, Wang N, Hoerster G, Hastings C, Cho MJ, Scelonge C, Lenderts B, Chamberlin M, Cushatt J, et al. Morphogenic regulators Baby boom and Wuschel improve monocot transformation. Plant Cell. 2016;28(9):1998-2015.

24. Li A, Jia S, Yobi A, Geng Z, Sato SJ, Zhang C, Angelovici R, Clemente TE, Holding DR. Editing of an alpha-kafirin gene family increases, digestibility and protein quality in sorghum. Plant Physiol. 2018;177(4):1425-38.

25. Feng J, Jia W, Lv S, Bao H, Miao F, Zhang X, Wang J, Li J, Li D, Zhu C, Li S, Li Y. Comparative transcriptome combined with morphophysiological analyses revealed key factors for differential cadmium accumulation in two contrasting sweet sorghum genotypes. Plant Biotechnol J. 2018;16(2):558-71.

26. McKinley B, Rooney W, Wilkerson C, Mullet J. Dynamics of biomass partitioning, stem gene expression, cell wall biosynthesis, and sucrose accumulation during development of Sorghum bicolor. Plant J. 2016:88(4):662-80.

27. Murray SC, Rooney W, Mitchell S, Sharma A, Klein P, Mullet J, Kresovich S. Genetic improvement of sorghum as a biofuel feedstock: II. QTL for stem and leaf structural carbohydrates. Crop Sci. 2008;48(6):2180-93.

28. Burks PS, Kaiser CM, Hawkins EM, Brown PJ. Genome wide association for sugar yield in sweet sorghum. Crop Sci. 2015;55(5):2138-48.

29. Swanson AF, Parker JH. Inheritance of smut resistance and juiciness of stalk in the sorghum cross, Red Amber $\times$ feterita. J Hered. 1931;22:51-6.

30. Casto A, McKinley B, Yu K, Rooney W, Mullet J. Sorghum stem aerenchyma formation is regulated by SbNAC_D during internode development. Plant Direct. 2018:2:1-16.

31. Fujimoto M, Sazuka T, Oda Y, Kawahigashi H, Wu J, Takanashi H, Ohnishi T, Yoneda J, Ishimori M, Kajiya-Kanegae H, et al. Transcriptional switch for programmed cell death in pith parenchyma of sorghum stems. Proc Natl Acad Sci USA. 2018;115(37):E8783-92.

32. Xia J, Zhao Y, Burks P, Pauly M, Brown P. A sorghum NAC gene is associated with variation in biomass properties and yield potential. Plant Direct. 2018;2:1-11.

33. Zhang LM, Leng CY, Luo H, Wu XY, Liu ZQ, Zhang YM, Zhang $H$, Xia Y, Shang L, Liu CM, et al. Sweet sorghum originated through selection of Dry, a plant-specific NAC transcription factor gene. Plant Cell. 2018:30(10):2286-307.

34. Mace ES, Innes D, Hunt C, Wang X, Tao Y, Baxter J, Hassall M, Hathorn A, Jordan D. The Sorghum QTL Atlas: a powerful tool for trait dissection, comparative genomics and crop improvement. Theor Appl Genet. 2019;132(3):751-66

35. Brenton Z, Cooper E, Myers M, Boyles R, Shakoor N, Zielinski K, Rauh $B$, et al. A genomic resource for the development, improvement, and exploitation of sorghum for bioenergy. Genetics. 2016;204(1):21-33.

36. Guan Y, Wang H, Qin L, Zhang H, Yang Y, Gao F, Li R, et al. QTL mapping of bio-energy related traits in Sorghum. Euphytica. 2011:182:431-40.

37. Shiringani AL, Frisch M, Friedt W. Genetic mapping of QTLs for sugarrelated traits in a RIL population of Sorghum bicolor L. Moench. Theor Appl Genet. 2010;121(2):323-36.

38. Ritter KB, Jordan DR, Chapman SC, Godwin ID, Mace ES, Lynne Mclntyre C. Identification of QTL for sugar-related traits in a sweet $\times$ grain sorghum (Sorghum bicolor L. Moench) recombinant inbred population. Mol Breed. 2008;22(3):367-84.

39. Natoli A, Gorni C, Chegdani F, Ajmone Marsan P, Colombi C, Lorenzon C, Marocco A. Identification of QTLs associated with sweet sorghum quality. Maydica. 2002;47(3-4):311-22.

40. Tarpley L, Vietor DM, Miller FR. Metabolism of sucrose during storage in intact sorghum stalk. Intl J Plant Sci. 1996;157:159-63.

41. Tarpley L, Vietor DM. Compartmentation of sucrose during radial transfer in mature sorghum culm. BMC Plant Biol. 2007:7:33.

42. Bihmidine S, Baker RF, Hoffner C, Braun DM. Sucrose accumulation in sweet sorghum stems occurs by apoplasmic phloem unloading and does not involve differential Sucrose transporter expression. BMC Plant Biol. 2015;15:186.

43. Milne RJ, Offler CE, Patrick JW, Crof CP. Cellular pathways of source leaf phloem loading and phloem unloading in developing stems of Sorghum bicolor in relation to stem sucrose storage. Funct Plant Biol. 2015;42(10):957-70.

44. Milne RJ, Byrt CS, Patrick JW, Grof CP. Are sucrose transporter expression profiles linked with patterns of biomass partitioning in Sorghum phenotypes? Front Plant Sci. 2013;4:223.

45. Mizuno H, Kasuga S, Kawahigashi $H$. The sorghum SWEET gene family: stem sucrose accumulation as revealed through transcriptome profiling. Biotechnol Biofuels. 2016;9:127.

46. Bihmidine S, Julius BT, Dweikat I, Braun DM. Tonoplast Sugar Transporters SbTSTs. putatively control sucrose accumulation in sweet sorghum stems. Plant Signal Behav. 2016;11:e1117721.

47. Eom JS, Chen LQ, Sosso D, Julius BT, Lin I, Qu XQ, Braun D, Frommer WB. SWEETs, transporters for intracellular and intercellular sugar translocation. Curr Opin Plant Biol. 2015;25:53-62

48. Chen LQ, Qu XQ, Hou BH, Sosso D, OsoRio S, Fernie AR, Frommer WB. Sucrose efflux mediated by SWEET proteins as a key step for phloem transport. Science. 2012;335(6065):207-11.

49. Chardon F, Bedu M, Calenge F, Klemens P, Spinner L, Clement G, Chietera G, Leran S, Ferrand M, Lacombe B, et al. Leaf fructose content is controlled by the vacuolar transporter SWEET17 in Arabidopsis. Curr Biol. 2013;23(8):697-702.

50. Lin IW, Sosso D, Chen LQ, Gase K, Kim SG, Kessler D, Klinkenberg PM, Gorder MK, Hou BH, Qu XQ, et al. Nectar secretion requires sucrose phosphate synthases and the sugar transporter SWEET9. Nature. 2014;508(7497):546-9.

51. Li Y, Feng S, Ma S, Sui X, Zhang Z. Spatiotemporal expression and substrate specificity analysis of the cucumber SWEET gene family. Front Plant Sci. 2017;8:1855.

52. Sosso D, Luo D, Li QB, Sasse J, Yang J, Gendrot G, Suzuki M, Koch KE, McCarty DR, Chourey PS, et al. Seed filling in domesticated maize and rice depends on SWEET-mediated hexose transport. Nat Genet. 2015;47:1489-96.

53. Bezrutczyk M, Hartwig T, Horschman M, Char SN, Yang J, Yang B, Frommer WB, Sosso D. Impaired phloem loading in zmsweet13a,b,c sucrose transporter triple knock-out mutants in Zea mays. New Phytol. 2018;218(2):594-603.

54. Yang J, Luo D, Yang B, Frommer WB, Eom JS. SWEET11 and 15 as key players in seed filling in rice. New Phytol. 2018:218(2):604-15.

55. Streb S, Zeeman SC. Starch metabolism in Arabidopsis. Arabidopsis Book. 2010;10:e0160.

56. Hannah LC, Boehlein S. Starch biosynthesis in maize endosperm. In: Larkins BA, editor. Maize Kernel development. Wallingford: CABI International; 2017. p. 149-59.

57. Kunz HH, Hausler RE, Fettke J, Herbst K, Niewiadomski P, Gierth M, Bell K, Steup M, Flugge UI, Schneider A. The role of plastidial glucose6-phosphate/phosphate translocators in vegetative tissues of Arabidopsis thaliana mutants impaired in starch biosynthesis. Plant Biol. 2010;12(S1):115-28.

58. Critchley JH, Zeeman SC, Takaha T, Smith AM, Smith SM. A critical role for disproportionating enzyme in starch breakdown is revealed by a knock-out mutation in Arabidopsis. Plant J. 2001;26(1):89-100.

59. Delatte T, Umhang M, Trevisan M, Eicke S, Thorneycroft D, Smith SM, Zeeman SC. Evidence for distinct mechanisms of starch granule breakdown in plants. J Biol Chem. 2006;281(17):12050-9. 
60. Ritte G, Heydenreich M, Mahlow S, Haebel S, Kotting O, Steup M. Phosphorylation of C6- and C3-positions of glucosyl residues in starch is catalyzed by distinct dikinases. FEBS Lett. 2006;580(20):4872-6.

61. Tanaka K, Murata K, Yamazaki M, Onosato K, Miyao A, Hirochika H. Three distinct rice cellulose synthase catalytic subunit genes required for cellulose synthesis in the secondary cell wall. Plant Physiol. 2003;133(1):73-83.

62. Persson S, Paredez A, Carroll A, Palsdottir H, Doblin M, Poindexter P, Khitrov N, Auer M, Somerville CR. Genetic evidence for three unique components in primary cell wall cellulose synthase complexes in Arabidopsis. Proc Natl Acad Sci USA. 2007;104(39):15566-71.

63. Little A, Schwerdt JG, Shirley NJ, Khor SF, Neumann K, O'Donovan LA, Lahnstein J, Collins HM, Henderson M, Fincher GE, et al. Revised phylogeny of the cellulose synthase gene superfamily: insights into cell wall evolution. Plant Physiol. 2018;177(3):1124-41.

64. Burton RA, Gidley MJ, Fincher GB. Heterogeneity in the chemistry, structure and function of plant cell walls. Nat Chem Biol. 2010;6:724-32.

65. Xu Z, Zhang D, Hu J, Zhou X, Ye X, Reichel KL, Stewart NR, Syrenne RD, Yang $X$, Gao P, et al. Comparative genome analysis of lignin biosynthesis gene families across the plant kingdom. BMC Bioinform. 2009;10:S3.

66. Sattler SE, Saballos A, Xin Z, Funnell-Harris DL, Vermerris W, Pedersen JF. Characterization of novel sorghum brown midrib mutants from an EMS-mutagenized population. G3 (Bethesda). 2014;4(11):2115-24.

67. Zheng L, Guo X, He B, Sun L, Peng Y, Dong S, Liu T, et al. Genome-wide patterns of genetic variation in sweet and grain sorghum (Sorghum bicolor). Genome Biol. 2011;12:R114.

68. Mace ES, Tai S, Gilding EK, Li Y, Prentis PJ, Bian L, Campbell BC, Hu W, Innes DJ, Han X. Whole-genome sequencing reveals untapped genetic potential in Africa's indigenous cereal crop sorghum. Nat Commun. 2013:4:2320.

69. Calvino M, Bruggmann R, Messing J. Screen of genes linked to high-sugar content in stems by comparative genomics. Rice. 2008;1 (2):166-76.

70. Calvino M, Bruggmann R, Messing J. Characterization of the small RNA component of the transcriptome from grain and sweet sorghum stems. BMC Genomics. 2013;12:356.

71. Jiang $S, M a Z$ Z, Vanitha J, Ramachandran S. Genetic variation and expression diversity between grain and sweet sorghum lines. BMC Genomics. 2013;14:18.

72. Yu H, Cong L, Zhu Z, Wang C, Zou J, Tao C, Shi Z, et al. Identification of differentially expressed microRNA in the stems and leaves during sugar accumulation in sweet sorghum. Gene. 2015;571(2):221-30.

73. Broadhead DM. Registration of Rio sweet sorghum. Crop Sci. 1972;12(1):716.

74. Harrison RL, Miller FR. Registration of della sweet Sorghum. Crop Sci. 1993:33(6):1416.

75. Li Y, Mehta R, Messing J. A new high throughput assay for determining soluble sugar in sorghum internode-extracted juice. Planta. 2018;248(4):785-93.

76. Ritter K, Chapman S, Jordan D, Godwin I, McIntyre L. Investigating the use of sweet sorghum as a model for sugar accumulation in sugarcane. In: 4th international crop science congress, Brisbane, Australia. 2004.

77. Kawahigashi H, Kasuga S, Okuizumi H, Hiradate S, Yonemaru J. Evaluation of Brix and sugar content in stem juice from sorghum varieties. Grassland Sci. 2013;59:11-9.

78. Trapnell C, Roberts A, Goff L, Pertea G, Kim D, Kelley DR, Pimentel H, et al. Differential gene and transcript expression analysis of RNA-seq experiments with TopHat and Cufflinks. Nat Protoc. 2012;7:562-79.

79. Anders S, Pyl PT, Huber W. HTSeq-a Python framework to work with high-throughput sequencing data. Bioinformatics. 2015;31(2):166-9.

80. McCarthy DJ, Chen Y, Smyth GK. Differential expression analysis of multifactor RNA-Seq experiments with respect to biological variation. Nucleic Acids Res. 2012:40(10):4288-97.

81. Sun Z, Chai HS, Wu Y, White WM, Donkena KV, Klein CJ, Garovic VD, Therneau TM, Kocher JP. Batch effect correction for genome-wide methylation data with Illumina Infinium platform. BMC Med Genomics. 2011:4:84.

82. Bolstad B. preprocessCore: a collection of pre-processing functions. R package version 1.44.0. 2018. https://github.com/bmbolstad/prepr ocessCore. Accessed 1 Sept 2018.
83. Carpita NC, Tierney M, Campbell M. Molecular biology of the plant cell wall: searching for the genes that define structure, architecture and dynamics. Plant Mol Biol. 2001:47(1-2):1-5.

84. Makita Y, Shimada S, Kawashima M, Kondou-Kuriyama T, Toyoda T, Matsui M. MOROKOSHI: transcriptome database in Sorghum bicolor. Plant Cell Physiol. 2015;56(1):e6.

85. Usadel B, Poree F, Nagel A, Lohse M, Czedik-Eysenberg A, Stitt M. A guide to using MapMan to visualize and compare omics data in plants: a case study in the crop species, Maize. Plant Cell Environ. 2009;32(9):1211-29.

86. Friso G, Majeran W, Huang M, Sun Q, van Wijk KJ. Reconstruction of metabolic pathways, protein expression, and homeostasis machineries across maize bundle sheath and mesophyll chloroplasts: large-scale quantitative proteomics using the first maize genome assembly. Plant Physiol. 2010;152(3):1219-50.

87. Chae L, Kim T, Nilo-Poyanco R, Rhee SY. Genomic signatures of specialized metabolism in plants. Science. 2014;344(6183):510-3.

88. Kanehisa M, Sato Y, Kawashima M, Furumichi M, Tanabe M. KEGG as a reference resource for gene and protein annotation. Nucleic Acid Res. 2015;44(D1):D457-62.

89. Campbell BC, Gilding EK, Mace ES, Tai S, Tao Y, Prentis PJ, Thomelin P, et al. Domestication and the storage starch biosynthesis pathway: signatures of selection from a whole sorghum genome sequencing strategy. Plant Biotechnol J. 2016;14(12):2240-53.

90. Kumar S, Stecher G, Tamura K. MEGA7: molecular evolutionary genetics analysis version 7.0 for bigger datasets. Mol Biol Evol. 2016;33(7):1870-4.

91. Dimitroff G, Little A, Lahnstein J, Schwerdt JG, Srivastava V, Bulone V, Burton RA, Fincher GB. $(1,3 ; 1,4)-\beta$-Glucan biosynthesis by the CSLF6 enzyme: position and flexibility of catalytic residues influence product fine structure. Biochemistry. 2016;55(16):2054-61.

92. Rai KM, Thu SW, Balasubramanian VK, Cobos CJ, Disasa T, Mendu V. Identification, characterization, and expression analysis of cell wall related genes in Sorghum bicolor L. Moench, a food, fodder, and biofuel crop. Front Plant Sci. 2016;7:1287.

93. Goubet F, Barton CJ, Mortimer JC, Yu X, Zhang Z, Miles GP, Richens J, Liepman AH, Seffen K, Dupree P. Cell wall glucomannan in Arabidopsis is synthesised by CSLA glycosyltransferases, and influences the progression of embryogenesis. Plant J. 2009;60(3):527-38.

94. Rips S, Bentley N, Jeong IS, Welch JL, von Schaewen A, Koiwa H. Multiple $\mathrm{N}$-Glycans cooperate in the subcellular targeting and functioning of Arabidopsis KORRIGAN1. Plant Cell. 2014;26(9):3792-808.

95. Mansoori N, Timmers J, Desprez T, Kamei CL, Dees DC, Vincken JP, Visser RG, Höfte H, Vernhettes S, Trindade LM. KORRIGAN1 interacts specifically with integral components of the cellulose synthase machinery. PLOS ONE. 2014;10(10):e0140411.

96. Glass M, Barkwill S, Unda F, Mansfield SD. Endo- $\beta-1,4-$ glucanases impact plant cell wall development by influencing cellulose crystallization. J Integr Plant Biol. 2015;57(4):396-410.

97. Amaducci S, Monti A, Venturi G. Non-structural carbohydrates and fibre components in sweet and fibre sorghum as affected by low and normal input techniques. Ind Crop Prod. 2004;20(1):111-8.

98. Byrt CS, Betts NS, Tan HT, Lim WL, Ermawar RA, Nguyen HY, et al. Prospecting for energy-rich renewable raw materials: sorghum stem case study. PLoS ONE. 2016;11:e0156638.

99. Saballos A, Sattler SE, Sanchez E, Foster TP, Xin Z, Kang C, Pedersen JF, et al. Brown midrib2 (Bmr2) encodes the major 4-coumarate: coenzyme A ligase involved in lignin biosynthesis in sorghum (Sorghum bicolor (L.) Moench). Plant J. 2012;70(5):818-30.

100. Jun SY, Sattler SA, Cortez GS, Vermerris W, Sattler SE, Kang C. Biochemical and structural analysis of substrate specificity of a phenylalanine ammonia-lyase. Plant Physiol. 2018;176(2):1452-68.

101. Saballos A, Ejeta G, Sanchez E, Kang C, Vermerris W. A Genome wide analysis of the cinnamyl alcohol dehydrogenase family in sorghum [Sorghum bicolor (L.) Moench] identifies SbCAD2 as the brown midrib6 gene. Genetics. 2009;181(2):783-95.

102. Barakat A, Yassin NB, Park JS, Choi A, Herr J, Carlson JE. Comparative and phylogenomic analyses of cinnamoyl-CoA reductase and cinnamoyl-CoA-reductase-like gene family in land plants. Plant Sci. 2011;181(3):249-57. 
103. Walker AM, Hayes RP, Youn B, Vermerris W, Sattler SE, Kang C. Elucidation of the structure and reaction mechanism of Sorghum hydroxycinnamoyltransferase and its structural relationship to other coenzyme A-dependent transferases and synthases. Plant Physiol. 2013;162(2):640-51.

104. Walker AM, Sattler SA, Regner M, Jones JP, Ralph J, Vermerris W, Sattler $\mathrm{SE}$, Kang C. The structure and catalytic mechanism of Sorghum bicolor caffeoyl-CoA O-methyltransferase. Plant Physiol. 2016;172(1):78-92.

105. Green AR, Lewis KM, Barr JT, Jones JP, Lu F, Ralph J, Vermerris W, Sattler $\mathrm{SE}$, Kang C. Determination of the structure and catalytic mechanism of Sorghum bicolor caffeic acid O-methyltransferase and the structural impact of three brown midrib12 mutations. Plant Physiol. 2014;165(4):1440-56.

106. Jun SY, Walker AM, Kim H, Ralph J, Vermerris W, Sattler SE, Kang C. The enzyme activity and substrate specificity of two major cinnamyl alcohol dehydrogenases in sorghum Sorghum bicolor., SbCAD2 and SbCAD4. Plant Physiol. 2017;174(4):2128-45.

107. Sattler SA, Walker AM, Vermerris W, Sattler SE, Kang C. Structural and biochemical characterization of cinnamoyl-CoA reductases. Plant Physiol. 2017;173(2):1031-44.

108. Moffatt BA, Weretilnyl EA. Sustaining S-adenosyl-L-methioninedependent methyltransferase activity in plant cells. Physiol Plant. 2001;113:435-42.

109. Dyson BC, Webster RE, Johnson GN. GPT2: a glucose 6-phosphate/ phosphate translocator with a novel role in the regulation of sugar signaling during seedling development. Ann Bot. 2014;113(4):643-52.

110. Dyson BC, Allwood JW, Feil R, Xu Y, Miller M, Bowsher CG, Goodacre R, et al. Acclimation of metabolism to light in Arabidopsis thaliana: the glucose 6-phosphate/phosphate translocator GPT2 directs metabolic acclimation. Plant Cell Environ. 2015;38(7):1404-17.

111. Juarez-Colunga S, Lopez-Gonzalez C, Morales-Elias NC, MassangeSanchez JA, Trachsel S, Tiessen A. Genome-wide analysis of the invertase gene family from maize. Planta. 2018;97(4-5):385-406.

112. Wang L, Zheng Y, Ding S, Zhang Q, Chen Y, Zhang J. Molecular cloning, structure, phylogeny, and expression analysis of the invertase gene family in sugarcane. BMC Genomics. 2017;17(1):109.

113. Lingle SE. Sucrose metabolism in the primary culm of sweet sorghum during development. Crop Sci. 1987;27(6):1214-9.

114. Figueroa CM, Lunn JE. A tale of two sugars: trehalose 6-phosphate and sucrose. Plant Physiol. 2016;172:7-27.

115. Chong J, Piron MC, Meyer S, Merdinoglu D, Bertsch C, Mestre P. The SWEET family of sugar transporters in grapevine: VVSWEET4 is involved in the interaction with Botrytis cinerea. J Exp Bot. 2014;65(22):6589-601.

116. Hu W, Hua X, Zhang Q, Wang J, Shen Q, Zhang X, Wang K, et al. New insights into the evolution and functional divergence of the SWEET family in Saccharum based on comparative genomics. BMC Plant Biol. 2018;18(1):270.
117. Wormit A, Trentmann O, Feifer I, Lohr C, Tjaden J, Meyer S, Schmidt U, Martinoia E, Neuhaus HE. Molecular identification and physiological characterization of a novel monosaccharide transporter from Arabidopsis involved in vacuolar sugar transport. Plant Cell. 2006;18(12):3476-90.

118. Jung B, Ludewig F, Schulz A, Meibner G, Wostefeld N, Flugge U, Pommerrenig B, Wirsching P, Sauer N, Koch W, et al. Identification of the transporter responsible for sucrose accumulation in sugar beet taproots. Nat Plants. 2015;1:14001.

119. Cheng J, Wen S, Xiao S, Lu B, Ma M, Bie Z. Overexpression of the tonoplast sugar transporter CmTST2 in melon fruit increases sugar accumulation. J Exp Bot. 2018;69(3):511-23.

120. Ren Y, Guo S, Zhang J, He H, Sun H, Tian S, Gong G, Zhang H, Levi A, Tadmor $Y$, et al. A tonoplast sugar transporter underlies a sugar accumulation QTL in watermelon. Plant Physiol. 2018;176(1):836-50.

121. Hedrich R, Sauer N, Neuhaus HE. Sugar transport across the plant vacuolar membrane: nature and regulation of carrier proteins. Curr Opin Plant Biol. 2015;25:63-70.

122. Hannah LC, Giroux M, Boyer C. Biotechnological modification of carbohydrates for sweet corns and maize development. Sci Hortic. 1993;55(1-2):177-97.

123. Nuccio ML, Wu J, Mowers R, Zhou HP, Meghji M, Primavesi LF, Paul M, et al. Expression of trehalose-6-phosphate phosphatase in maize ears improves yield in well-watered and drought conditions. Nat Biotechnol. 2015;33(8):862-74.

124. Oszvald M, Primavesi LF, Griffiths CA, Cohn J, Basu SS, Nuccio ML, Paul MJ. Trehalose 6-phosphate regulates photosynthesis and assimilate partitioning in reproductive tissue. Plant Physiol. 2018;176(4):2623-38.

125. Godin B, Nagle N, Sattler S, Agneessens R, Delcarte J, Wolfrum E. Improved sugar yields from biomass sorghum feedstocks: comparing low-lignin mutants and pretreatment chemistries. Biotechnol Biofuels. 2016;9:251

126. Rivera-Burgos LA. Genetic, agronomic and compositional characterization of brown midrib sweet sorghum lignocellulosic biomass for ethanol production. 2015. Purdue University.

127. Fernie AR, Stitt M. On the discordance of metabolomics with proteomics and transcriptomics: coping with increasing complexity in logic, chemistry, and network interactions. Plant Physiol. 2012;158:1139-45.

128. Schwender J, Konig C, Klapperstuck M, Heinzel N, Munz E, Hebelmann I, Hay J, et al. Transcript abundance on its own cannot be used to infer fluxes in central metabolism. Front Plant Sci. 2014;5:668.

\section{Publisher's Note}

Springer Nature remains neutral with regard to jurisdictional claims in published maps and institutional affiliations.
Ready to submit your research? Choose BMC and benefit from:

- fast, convenient online submission

- thorough peer review by experienced researchers in your field

- rapid publication on acceptance

- support for research data, including large and complex data types

- gold Open Access which fosters wider collaboration and increased citations

- maximum visibility for your research: over 100M website views per year

At BMC, research is always in progress.

Learn more biomedcentral.com/submissions 